\title{
Thermodynamics of Transport Through the Ammonium Transporter Amt-1 Investigated with Free Energy Calculations
}

\author{
R. Thomas Ullmann, ${ }^{*}{ }^{\dagger}$ Susana L. A. Andrade, ${ }^{*}{ }^{\ddagger}$ and G. Matthias Ullmann ${ }^{*}{ }^{\dagger}$ \\ ${ }^{\dagger}$ Structural Biology/Bioinformatics, University of Bayreuth, Universitätsstrasse 30, BGI, 95447 Bayreuth, Germany \\ ${ }^{\ddagger}$ Institute of Organic Chemistry and Biochemistry and BIOSS Centre for Biological Signalling Studies, Albert-Ludwigs-Universität \\ Freiburg, Albertstrasse 21, 79104 Freiburg, Germany
}

Supporting Information

ABSTRACT: Amt-1 from Archaeoglobus fulgidus (AfAmt-1) belongs to the Amt/Rh family of ammonium/ammonia transporting membrane proteins. The transport mode and the precise microscopic permeation mechanism utilized by these proteins are intensely debated. Open questions concern the identity of the transported substrate (ammonia and/or ammonium) and whether the transport is passive or active. To address these questions, we studied the overall thermodynamics of the different transport modes as a function of the environmental conditions. Then, we investigated the thermodynamics of the underlying microscopic

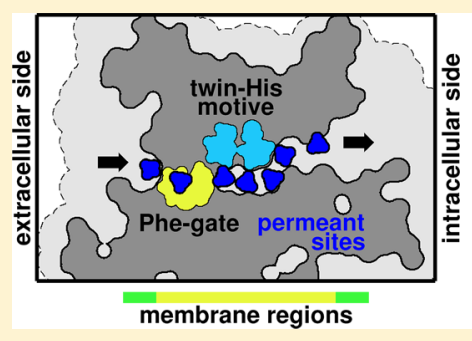
thermodynamics of possible permeation mechanisms investigated with free energy calculations

electroneutral $\mathrm{NH}_{3}$ uniport electrogenic $\mathrm{NH}_{4}^{+}$uniport electrogenic $\mathrm{NH}_{3} / \mathrm{H}^{+}$symport transport mechanisms with free energy calculations within a continuum electrostatics model. The formalism developed for this purpose is of general utility in the calculation of binding free energies for ligands with multiple protonation forms or other binding forms. The results of our calculations are compared to the available experimental and theoretical data on Amt/Rh proteins and discussed in light of the current knowledge on the physiological conditions experienced by microorganisms and plants. We found that microscopic models of electroneutral and electrogenic transport modes are in principle thermodynamically viable. However, only the electrogenic variants have a net thermodynamic driving force under the physiological conditions experienced by microorganisms and plants. Thus, the transport mechanism of AfAmt-1 is most likely electrogenic.

\section{INTRODUCTION}

Amt-1 from Archaeoglobus fulgidus (AfAmt-1) belongs to the widespread family of Amt/Rh transmembrane transport proteins. The ammonium transport (Amt) proteins supply microorganisms and plants with ammonium as the most directly utilizable nitrogen source. ${ }^{1-11}$ The human Rhesus ( $\mathrm{Rh}$ ) proteins are implicated in ammonium excretion and $\mathrm{pH}$ regulation functions. ${ }^{11-13}$

The precise molecular mechanism of substrate conduction through Amt/Rh proteins is not yet known. It is debated whether the uncharged ammonia or the charged ammonium ion is the species that is actually translocated by the proteins. $^{2-11,14}$ Traditionally, it was thought that the transported species is $\mathrm{NH}_{4}{ }^{+1}$. This opinion was grounded on several lines of evidence. First, the $\mathrm{p} K_{\mathrm{a}}$ value of 9.25 makes $\mathrm{NH}_{4}{ }^{+}$much more abundant than $\mathrm{NH}_{3}$ at physiological $\mathrm{pH}$ values around 7.0. Second, lipid membranes possess a significant permeability for the apolar $\mathrm{NH}_{3}$ molecule, which seemed to make a dedicated transport protein unnecessary. Third and possibly most important, the charge carried by the ammonium ion makes it possible to drive its electrogenic transmembrane transport by the electric transmembrane potential. This possibility would enable a cell to take up ammonium also at very low concentrations of ammonium in the extracellular medium and to compensate the loss of ammonia through unspecific permeation of the cell membrane in a process called cyclic retention. ${ }^{1}$

The assumption of an active transport mechanism was called into question by some physiological experiments whose interpretation led to the hypothesis that $\mathrm{Amt} / \mathrm{Rh}$ proteins actually facilitate the passive, electroneutral transport of the uncharged ammonia molecule. ${ }^{11,15,16}$ These experiments were supported by the first X-ray crystal structures of AmtB from Escherichia coli $(\mathrm{EcAmtB})$ that revealed a transmembrane pore with a hydrophobic central region whose permeation by the charged ammonium ion was considered to be thermodynamically prohibitive. ${ }^{8,11,16,17}$ According to this hypothesis, $\mathrm{NH}_{4}^{+}$ would be recruited at the extracellular side leaving the proton behind in the extracellular phase. The resulting ammonia molecule would then permeate the transmembrane pore and be reprotonated at the intracellular side.

However, as noted in our earlier work, an electroneutral uniport of $\mathrm{NH}_{3}$ poses the bioenergetic problem of how a cell can retrieve ammonia/ammonium at low extracellular concentrations of the substrate. ${ }^{4,18}$ Reports of membrane potential dependent uptake currents in plant and microbial Amts support the view of an electrogenic transport that would involve $\mathrm{NH}_{4}^{+}$

Received: June 3, 2012

Revised: July 13, 2012

Published: July 17, 2012 
either in an uniport of $\mathrm{NH}_{4}{ }^{+}$or a symport of $\mathrm{NH}_{3}$ and $\mathrm{H}^{+} .{ }^{19-27}$ A membrane potential dependence of the ammonium uptake activity was also reported for Rh type transporters, ${ }^{28-30}$ but partly discarded as too weak to be significant. ${ }^{28,29}$ The finding that the transport activity of the $\mathrm{Rh}$ proteins was stimulated by an extracellular alkalinization led to the interpretation that $\mathrm{NH}_{3}$ must be involved in either a uniport of $\mathrm{NH}_{3}{ }^{11,13,28,29}$ or a symport of $\mathrm{NH}_{3}$ and $\mathrm{NH}_{4}+{ }^{+30}$ A summarizing scheme of the possible transport mechanisms considered to date ${ }^{4,5,31}$ is shown in Figure 1. ${ }^{4}$

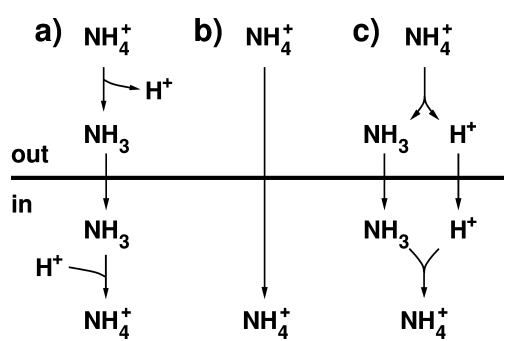

Figure 1. Mechanistic scheme of transport modes that could be operative in AfAmt-1. ${ }^{4}$ (a) At physiological $\mathrm{pH}$ values ammonium will be almost exclusively in the cationic form, such that translocation of $\mathrm{NH}_{3}$ implies extracellular deprotonation and intracellular reprotonation. Electrogenic transport of ammonium is either a net uniport of $\mathrm{NH}_{4}{ }^{+}$as depicted in panel $\mathrm{b}$ or a symport of $\mathrm{NH}_{3} / \mathrm{H}^{+}$as depicted in panel c. Both electrogenic transport modes result in a net transport of $\mathrm{NH}_{4}{ }^{+}$. During a uniport the ammonium ion is translocated as an intact entity. In contrast, during a symport the ammonium ion is separated into an ammonia molecule and a proton, which may be translocated along different routes, and recombined on the inner side of the membrane.

Figure 2 shows the structure of one monomer within the AfAmt-1 trimer highlighting the transmembrane pore and putative intermediate sites passed by the permeant. ${ }^{18}$ The transmembrane pore can be subdivided into a central hydrophobic pore lumen and two wider and less hydrophobic vestibule regions at the intracellular and extracellular ends of the pore. The extracellular vestibule and the pore lumen are separated by a narrow constriction that is formed by two conserved phenylalanine residues (F96 and F204). The socalled Phe-gate has been attributed a role in the dehydration and subsequent deprotonation of the ammonium ion either directly at or close to the Phe-gate. ${ }^{5,32,33}$ The pore lumen is lined by hydrophobic residues except for a conserved pair of histidine residues ( $\mathrm{H} 157$ and $\mathrm{H} 305$ ) termed the twin-His motif. The twin-His residues are arranged in a nearly coplanar geometry, with their $\mathrm{N} \delta$ atoms within hydrogen-bonding distance. A role of the twin-His motif in the conduction mechanism has been suggested based on the structural data and mutagenesis experiments. This role could either be the providing of hydrogen bond interactions to the permeant ${ }^{16,34}$ or as transient proton acceptor in the deprotonation of the ammonium ion. $5,17,35$

Most previous theoretical studies of substrate permeation through Amt/Rh proteins concentrated on different aspects of substrate permeation through EcAmtB. ${ }^{14,32,33,36-49}$ One work studied the thermodynamics of substrate permeation through Rh50 from Nitrosomonas europaea (NeRh50). ${ }^{50}$ In this work, we studied the thermodynamics of the different possible transport mechanisms of AfAmt-1. We extend upon previous theoretical studies by including the effects of substrate

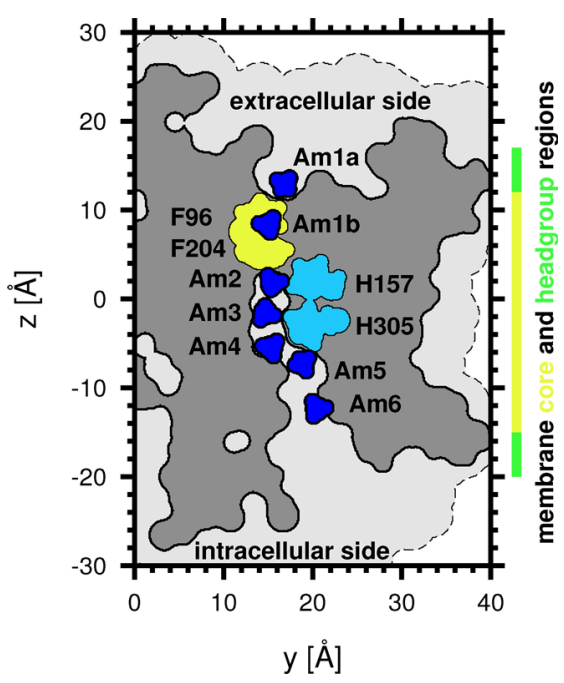

Figure 2. Structure of Amt-1 from Archaeoglobus fulgidus (AfAmt-1). The extracellular side is shown at the top and the intracellular side at the bottom. The figure shows a single monomer. The boundaries of the membrane core (yellow) and headgroup regions (green) are indicated by the bars on the right-hand side of the plot. The white regions at the top and the bottom denote the solvent phases. The light-gray outer region of Amt-1 is a projection of the solvent inaccessible volume of the transporter trimer into a plane perpendicular to the membrane. The darker inner region is a projection of a thin slice of Amt-1 into the same projection plane. The slice plane is slightly tilted with respect to the membrane normal to follow the course of the transmembrane pore. The positions of the investigated permeant positions (blue), the twin-histidine motif (cyan) and the Phe-gate (light yellow) are indicated.

concentration, electrochemical transmembrane gradients, proton-coupled binding equilibria, and competitive binding of different ligand species. Our free energy calculations are based on a microstate description of AfAmt-1 within a continuum electrostatics/molecular mechanics model. ${ }^{51,52}$ Such a description can provide valuable insight into biomolecular function and has already been successfully applied to other membrane proteins like bacteriorhodopsin, bacterial and plant photosynthetic reaction centers, cytochrome $b c_{1}$, quinol-fumarate reductase, and cytochrome $c$ oxidase. ${ }^{52-57}$

\section{METHODS}

Microstate Description of the Receptor-Ligand System. Our model is based on a microstate description of the system. ${ }^{51,58,59}$ A microstate is defined by a particular instance occupied by each site. The term instance describes the combination of a particular set of atomic partial charges, a particular number of bound ligands of each ligand type and a particular set of atomic coordinates. The energy of a microstate $\mathbf{n}$ is given by ${ }^{51}$

$$
E_{\mathbf{n}}^{\text {micro }}=\sum_{i=1}^{\mathrm{N}^{\text {sites }}}\left(E_{i, k}^{\text {int }, \mathrm{r}}-\sum_{m}^{\mathcal{L}} \nu_{i, k, m} \bar{\mu}_{m}\right)+\sum_{i=1}^{N^{\text {sites }}} \sum_{j=1}^{j<i} W_{i, k, j, l}
$$

where $N^{\text {sites }}$ is the number of sites and $\mathcal{L}$ is the number of ligand types. $E_{i, k}^{\text {int,r }}$ is the intrinsic energy of site $i$ in instance $k$, where the superscript $r$ designates the receptor environment. The stoichiometric coefficient $\nu_{i, k, m}$ indicates the number of ligands of type $m$ bound to instance $k$ of site $i$ and $\bar{\mu}_{m}$ is the electrochemical potential of this ligand in the adjacent solution. 
$W_{i, k, j, l}$ is the interaction energy of sites $i$ and $j$ in their respective instances $k$ and $l$.

The intrinsic energy in the receptor environment $E_{i, k}^{\text {int,r }}$ is calculated relative to the intrinsic energy of an appropriate model compound in solution $E_{i, k}^{\mathrm{int}, \mathrm{m} 60,61}$

$$
E_{i, k}^{\mathrm{int}, \mathrm{r}}=E_{i, k}^{\mathrm{int}, \mathrm{m}}+\Delta E_{i, k}^{\mathrm{int}}
$$

The intrinsic energy shift $\Delta E_{i, k}^{\text {int }}$ upon transfer from the solution to the receptor environment is obtained from a continuumelectrostatics/molecular mechanics model. ${ }^{51,52,62,63}$

The definition of an equilibrium state requires that each site binds its ligands exclusively from one of the membrane sides only and that no transfer of ligands takes place between the membrane sides. ${ }^{62,63}$ Thus, the connectivity of each site to one of the membrane sides has to be determined. In the transmembrane pore of AfAmt-1, the Phe-gate separates the membrane sides and thus defines the connectivity of the porelining residues. The permeant sites Amla and Amlb are connected to the extracellular side. The rest of the permeant sites and the twin-His residues are connected to the intracellular side. All other sites are assigned to either membrane side based on their exposure to either solvent phase or their involvement in hydrogen bonding networks (Table $S 1$ of the Supporting Information).

An additional complication arises if a ligand can undergo binding reactions with other ligands, e.g., if the ligand itself is protonatable. In such a case all of the binding equilibria involving the macromolecular receptor, the ligand, and the proton are coupled. The corresponding expressions for the ligand chemical potentials in our case are given below.

Electrochemical Potentials of the Ligands. The chemical potential of a ligand $i$ is given by

$$
\mu_{i}=\mu_{i}^{\circ}+\beta^{-1} \ln a_{i}
$$

where $\mu_{i}^{\circ}$ and $a_{i}$ are the standard chemical potential and the activity of the ligand, respectively. The electrochemical potential of the ligand $i$ extends the chemical potential by the energy of the ligand in the electrostatic potential of the surrounding solution

$$
\bar{\mu}_{i}=\mu_{i}+z_{i} \mathrm{~F} \Theta \Delta \psi
$$

where $z_{i}$ is the formal charge of the ligand, $F$ is the Faraday constant, and $\Delta \psi$ is the electric transmembrane potential. By convention, the Heaviside step function $\Theta$ adopts a value of 0 if the ligand resides in the extracellular phase and a value of 1 if the ligand resides the intracellular phase. ${ }^{64,65}$

In our case, there is a total of six different ligand species comprising the proton and the permeant species ammonia, ammonium, hydroxyl ion, water, and hydronium ion. The reaction equations for the corresponding protonation equilibria are given by

$$
\mathrm{NH}_{3}+\mathrm{H}^{+} \rightleftharpoons \mathrm{NH}_{4}^{+}
$$

and

$$
\mathrm{OH}^{-}+2 \mathrm{H}^{+} \rightleftharpoons \mathrm{H}_{2} \mathrm{O}+\mathrm{H}^{+} \rightleftharpoons \mathrm{H}_{3} \mathrm{O}^{+}
$$

The equilibrium condition demands that the sum of the electrochemical potentials of all species is equal for each stage of the protonation equilibria eqs 5 and 6 . Thus, we can write the electrochemical potential of each ligand species as the sum of the electrochemical potentials of the fully deprotonated ligand and of the protons bound by the species

$$
\begin{aligned}
& \bar{\mu}_{\mathrm{NH}_{4}}=\bar{\mu}_{\mathrm{NH}_{3}}+\bar{\mu}_{\mathrm{H}^{+}} \\
& \bar{\mu}_{\mathrm{H}_{2} \mathrm{O}}=\bar{\mu}_{\mathrm{OH}^{-}}+\bar{\mu}_{\mathrm{H}^{+}} \\
& \bar{\mu}_{\mathrm{H}_{3} \mathrm{O}^{+}}=\bar{\mu}_{\mathrm{OH}^{-}}+2 \bar{\mu}_{\mathrm{H}^{+}}
\end{aligned}
$$

Consequently, there are three independent ligand electrochemical potentials $\left(\bar{\mu}_{\mathrm{H}^{+}}, \bar{\mu}_{\mathrm{OH}^{-}}\right.$, and $\left.\bar{\mu}_{\mathrm{NH}_{3}}\right)$ for each membrane side.

The activity of the proton $a_{\mathrm{H}+}$ is given by the definition of the $\mathrm{pH}$ as

$$
a_{\mathrm{H}^{+}} \equiv 10^{-\mathrm{pH}}
$$

Similar to the $\mathrm{pH}$, we define the functions $\mathrm{pO}$ and $\mathrm{pN}$, as measures of the total activity of all water and ammonia protonation forms, respectively

$$
\begin{aligned}
& a_{\mathrm{NH}_{3}}+a_{\mathrm{NH}_{4}} \equiv 10^{-\mathrm{pN}} \\
& a_{\mathrm{OH}^{-}}+a_{\mathrm{H}_{2} \mathrm{O}}+a_{\mathrm{H}_{3} \mathrm{O}^{+}} \equiv 10^{-\mathrm{pO}}
\end{aligned}
$$

If we assume that the activity coefficients of all protonation forms are reasonably close to a value of $1.0, \mathrm{pN}$ and $\mathrm{pO}$ will be independent of the $\mathrm{pH}$ value. With this assumption, we can express $\mathrm{pN}$ and $\mathrm{pO}$ as functions of the total concentration of all ammonia and water species, respectively

$$
\begin{aligned}
& \mathrm{pN} \approx-\log _{10}\left[\frac{c_{\mathrm{NH}_{3}}+c_{\mathrm{NH}_{4}}^{+}}{c^{\circ}}\right] \\
& \mathrm{pO} \approx-\log _{10}\left[\frac{c_{\mathrm{OH}^{-}}+c_{\mathrm{H}_{2} \mathrm{O}}+c_{\mathrm{H}_{3} \mathrm{O}^{+}}}{c^{\circ}}\right]
\end{aligned}
$$

where the standard concentration is, by definition, given by $c^{\circ}$ $\equiv 1 \mathrm{~mol} / \mathrm{L}$. Our assumption should be fulfilled reasonably well under physiologically relevant conditions, i.e., for small concentrations of ammonia and ammonium $c_{\mathrm{NH}_{3}}+c_{\mathrm{NH}_{4}}{ }^{+} \ll 1$ $\mathrm{mol} / \mathrm{L}$ and $\mathrm{pH}$ values far from the extreme ends of the $\mathrm{pH}$ range. The activities of the individual protonation forms of ammonia and water can then be expressed as functions of $\mathrm{pN}$ and $\mathrm{pO}$. The activity of $\mathrm{NH}_{3}$ is given by

$$
a_{\mathrm{NH}_{3}}=\frac{10^{-\mathrm{pN}}}{1+\exp \left[-\beta\left(\mu_{\mathrm{NH}_{4}{ }^{+}}^{\circ}-\mu_{\mathrm{NH}_{3}}^{\circ}-\mu_{\mathrm{H}^{+}}\right)\right]}
$$

The activity of the $\mathrm{OH}^{-}$ion is given by

$$
\begin{aligned}
a_{\mathrm{OH}^{-}}= & 10^{-\mathrm{pO}} /\left\{1+\exp \left[-\beta\left(\mu_{\mathrm{H}_{2} \mathrm{O}}^{\circ}-\mu_{\mathrm{OH}^{-}}^{\circ}-\mu_{\mathrm{H}^{+}}\right)\right]\right. \\
& \left.+\exp \left[-\beta\left(\mu_{\mathrm{H}_{3} \mathrm{O}^{+}}^{\circ}-\mu_{\mathrm{OH}^{-}}^{\circ}-2 \mu_{\mathrm{H}^{+}}\right)\right]\right\}
\end{aligned}
$$

The total chemical potentials of the ammonia and the hydroxyl ion can be calculated via eq 3 . The chemical potentials of the other permeant species follow from eqs 7-9. A detailed derivation of eqs 15 and 16 can be found in section $A$ of the Supporting Information. Plots of the chemical potential of all permeant species as a function of the $\mathrm{pH}$ value of the solution can be found in Figure S1 of the Supporting Information. The standard chemical potentials of the permeant species are available from experiment (see Table S4 of the Supporting Information). In our calculations, we assume the total concentration of all water species to be fixed at $55.5 \mathrm{~mol} / \mathrm{L}$ as calculated from the density and the molecular weight of water $\left(\rho_{\mathrm{H}_{2} \mathrm{O}}=1 \mathrm{~kg} / \mathrm{L}, M_{\mathrm{H}_{2} \mathrm{O}}=18.015 \mathrm{~g} / \mathrm{mol}\right)$. 
Simulation Setup. Our simulations are based on the crystal structure of AfAmt-1 in the native form (PDB code $2 \mathrm{~B} 2 \mathrm{~F}){ }^{18}$ Hydrogen atoms were added with HBUILD ${ }^{66}$ in CHARMM $^{67}$ and their positions were subsequently energy minimized using the CHARMM force field. ${ }^{68}$ The intrinsic energies and interaction energies were computed from a continuum electrostatics/molecular mechanics model implemented in our program GCEM $^{69}$ based on our modified version of the MEAD library. ${ }^{55,70}$ All MC simulations were carried out with our program suite GMCT. ${ }^{51}$ Equilibrium probabilities of binding forms for individual sites were computed with the Metropolis MC method. ${ }^{71}$ Free energy calculations were performed with the free energy perturbation method ${ }^{72}$ in our recently presented generalization ${ }^{73}$ combined with the Bennett acceptance ratio method. ${ }^{74}$ Details of our computational method are specified in the remaining parts of this section.

Structure Preparation. The structure of AfAmt-1 in the native form (PDB code 2B2F) was used as basis for our calculations. ${ }^{18}$ Hydrogen atoms were added with HBUILD ${ }^{66}$ in $\mathrm{CHARMM}^{67}$ and their positions were subsequently energy minimized using the CHARMM force field as described below. ${ }^{68}$ Two additional rotamer positions were added for each hydroxyl or sulfhydryl hydrogen atom by varying the corresponding torsion angle in steps of $120^{\circ}$. Four alternative hydrogen positions for protonated forms of carboxylic acids were added to represent the syn and trans configurations of the dissociable proton at each carboxyl oxygen atom. The corresponding angles and bond lengths were taken from ref 69 .

The positions of the permeant sites (see Figure 2) were determined from putative binding sites obtained from experimental and theoretical studies at AfAmt- ${ }^{18}$ and the close homologue EcAmtB. ${ }^{16,17,32,33,48} \mathrm{Amla}$ corresponds to the ammonium recruitment site between $\mathrm{W} 137$ and S208 identified in the crystallographic studies. ${ }^{16-18,35}$ This recruitment site has also been verified in several independent $\mathrm{MD}$ studies of EcAmtB. ${ }^{32,33,36,37,39,41,44,47,48}$ Amlb was modeled based on observations in $\mathrm{MD}$ simulations of EcAmtB, where ammonium was sandwiched by the side chains of the two phenylalanine residues forming the Phe-gate. ${ }^{32,33,39,49}$ Similar conformations are also observed in computational studies of a complex formed by ammonium and two benzene molecules in aqueous solution. ${ }^{75}$ This binding site was proposed to promote dehydration of the ammonium ion and consequently to facilitate its deprotonation either directly at this site or at $\mathrm{Am} 2 .^{32,33,39} \mathrm{Am} 2$ was placed at the corresponding ammonia position proposed for EcAmtB (PDB 1U7G). ${ }^{16}$ Am3 and Am4 were placed at xenon binding sites as found in the xenonpressurized structure of AfAmt-1 (PDB 2B2J). ${ }^{18}$ These sites closely comply with the putative ammonia or water binding sites identified in the transmembrane pore lumen of AfAmt- $1^{18}$ and EcAmtB. ${ }^{16,17,35} \mathrm{Am} 5$ was placed at the position of a water molecule (water 414) found in PDB 2B2F directly below H305 at the lower end of the hydrophobic pore lumen. ${ }^{18}$ Am6 was modeled based on a proposed site of ammonia reprotonation in the intracellular vestibule of EcAmtB between the side chains of the residues that are equivalent to D300 and S263 of AfAmt$1 .^{16,32,33,48}$ The permeant positions were energy minimized while setting all atomic partial charges to zero to avoid biasing their protonation state. The positions of the permeant heavy atoms were constrained harmonically with a force constant of $0.5 \mathrm{kcal} /\left(\mathrm{mol} \AA^{2}\right)$ during the minimization. The coordinates of all hydrogen atoms were included in the energy minimization. All protein heavy atoms except for those of the side chains of
F96 and F204 were kept fixed during the minimization. Additional rotamers were added for all permeant sites by randomly rotating the initially generated coordinates in space resulting in an 8-fold increase in the number of rotamers.

Calculation of Intrinsic Energies and Interaction Energies. The intrinsic energies and interaction energies were computed from a continuum electrostatics/molecular mechanics model implemented in GCEM. ${ }^{76}$ We considered a single monomer explicitly with all titratable sites, whereas the other two monomers of the trimer are represented implicitly by their dielectric regions. All aspartate, histidine, glutamate, lysine, arginine, cysteine, and tyrosine residues and the termini were considered as protonatable sites. The permeant sites in the transmembrane pore were modeled with binding forms for each of the considered permeants and a ligand-free binding form as described above.

We used a detailed charge model with explicit hydrogen positions for all protonatable sites. Atomic partial charges for standard forms of amino-acid residues were taken from the CHARMM22 parameter set. $^{68}$ Atomic partial charges of nonstandard forms of amino acid residues were taken from ref 69. Model compounds of protonatable amino acids include the entire residue plus the directly neighboring CHARMM charge groups belonging to the backbone of the preceding and succeeding amino acids to ensure charge neutrality and to mimic an $\mathrm{N}$-formyl, $\mathrm{N}$-methylamide blocked amino acid compounds. $^{61}$ The intrinsic energies of the model compounds for protonatable amino acid residues in aqueous solution were calculated from $\mathrm{p} K_{\mathrm{a}}$ values of appropriate model compounds taken from the literature ${ }^{52,77}$ as described in ref 69. Atomic partial charges for the permeant sites were obtained from density functional theory calculations with the ADF program $^{78,79}$ (functionals VWN ${ }^{80}$ and BP86 ${ }^{81,82}$ with a QZ4P basis set). The atomic partial charges were calculated with the multipole derived charge analysis method ${ }^{83}$ (Table S2 of the Supporting Information). The intrinsic energies for the permeants in solution were computed from experimental data (Table S3 of the Supporting Information). The Lennard-Jones parameters of ammonia/ammonium were taken from the CHARMM22 force field (model compounds methylamine/ methylammonium). ${ }^{68}$ The Lennard-Jones parameters for the water species were taken from the TIP3P water model. ${ }^{84}$

Conformational energies of the sites were computed using the CHARMM22 force field, ${ }^{68}$ and added to the intrinsic energies. We used bonded terms involving atoms of the site, and Lennard-Jones interaction energies within the site and between the site and the background (i.e., parts of the protein not belonging to any site). Lennard-Jones interactions were also added to the site-site interaction energies.

MEAD uses a finite-difference method on cubic grids to solve the linearized Poisson-Boltzmann equation. ${ }^{85,86}$ The dielectric constant of the protein including the permeant sites was set to $4{ }^{55}$ The dielectric constant of the solvent including protein cavities and unoccupied space of the transmembrane pore was set to 80 . The position of the membrane was assigned according to the OPM database ${ }^{87}$ and visual inspection of PDB 2B2F. The dielectric constant of the membrane core, representing the hydrophobic lipid tails, was set to 2 and the dielectric constant of the polar headgroup region was set to 20 . The membrane core region extends from $z=-15$ to $+12 \AA$ in the coordinates defined by PDB $2 \mathrm{~B} 2 \mathrm{~F}$ (see Figure 2). The headgroup regions have a thickness of $5 \AA$. The temperature was set to $298.15 \mathrm{~K}$. The dielectric boundary between solute 

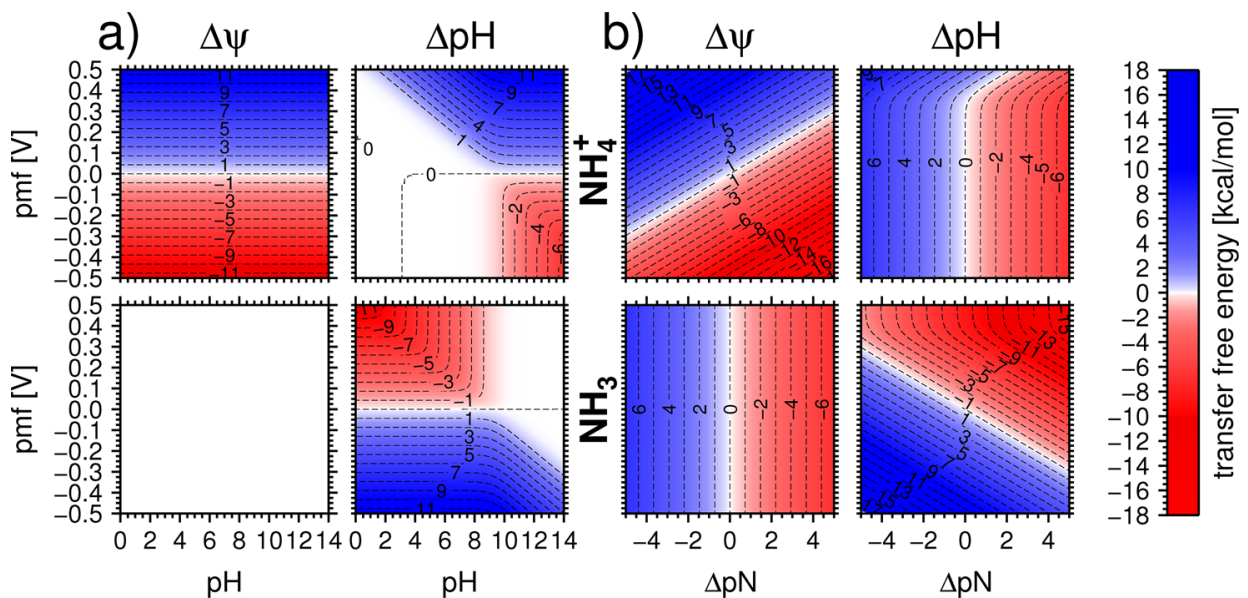

Figure 3. Transfer free energies for the import of ammonia (lower row) and ammonium (upper row). The transfer free energy is color coded (see color bar) and indicated by isocontours drawn in constant intervals of $1.0 \mathrm{kcal} / \mathrm{mol}$ (contour values given in $\mathrm{kcal} / \mathrm{mol}$ ). (a) Transfer free energies as a function of the intracellular $\mathrm{pH}$ and the proton-motive force. (b) Transfer free energies as a function of the transmembrane $\mathrm{pN}$ difference $(\Delta \mathrm{pN}=$ $\left.\mathrm{pN}^{\text {in }}-\mathrm{pN}^{\text {out }}\right)$ and the pmf. ( $\mathrm{a}$ and $\left.\mathrm{b}\right)$ The $\mathrm{pmf}$ in the left column consists entirely of an electric transmembrane potential $\left(\mathrm{pmf}=\psi^{\text {in }}-\psi^{\text {out }}\right)$. The $\mathrm{pmf}$ in the right column consists entirely of a transmembrane $\mathrm{pH}$ difference $(\mathrm{pmf} \approx-59 \mathrm{mV} \Delta \mathrm{pH})$.

and solvent was calculated using a water probe sphere of $1.4 \AA$ radius and the atomic radii ( $1.0 \AA$ for $\mathrm{H}, 1.55 \AA$ for $\mathrm{N}, 1.7 \AA$ for $\mathrm{C}, 1.5 \AA$ for $\mathrm{O}$, and $1.8 \AA$ for $\mathrm{S}$ ). The ionic strength was set to $0.15 \mathrm{M}$. The thickness of the ion exclusion layer was set to 2.0 A.

Electrostatic potentials were computed using the focusing technique $^{88}$ with four nested cubic grids. The grids for the computation of the electrostatic solvation and interaction energies had grid spacings of $2.0 \AA, 0.5 \AA, 0.2 \AA$ and $0.15 \AA$, respectively. The outer grid had a grid length of 101 points and was centered on the geometric center of the protein. The following grids were centered on the geometric center of the site. The second grid had a grid length of 241 points. The third grid had a grid length of 345 points. The inner grid had a grid length that was adjusted for each instance of each site separately to fit the dimensions of the site plus $15 \AA$ in each direction. The same grids were used for the model compound and the site in the protein. The grids for the computation of the electrostatic transmembrane potential ${ }^{64}$ had grid spacings of $2.0,0.5,0.35$, and $0.2 \AA$, respectively. The corresponding grid lengths were $121,373,387$, and 425 points. All grids were centered on the geometric center of the protein.

Monte Carlo Simulations. All MC simulations were carried out with our program suite GMCT. ${ }^{51}$ The temperature was set to $298.15 \mathrm{~K}$. The interaction energy cutoffs for pair and triplet moves were set to 1.0 and $2.0 \mathrm{kcal} / \mathrm{mol}$.

Equilibrium probabilities of binding forms for individual sites were computed with the Metropolis MC method. ${ }^{71,89,90}$ We used $5000 \mathrm{MC}$ scans for the equilibration and $10^{5} \mathrm{MC}$ scans for the production run.

Free energy calculations were performed with the free energy perturbation method ${ }^{72}$ in our recently presented generalization. ${ }^{73}$ We used staging ${ }^{91}$ with nine alchemical intermediate states evenly distributed along the transformation coordinate. The Bennett acceptance ratio method was used to minimize the statistical error of the free energy estimates. ${ }^{74}$ Each free energy calculation consisted of multiple simulations according to the random single-move simulation scheme. ${ }^{73}$ The number of simulations was increased until the statistical error of the free energy estimate was smaller than $0.01 \mathrm{kcal} / \mathrm{mol}$. Each separate simulation consisted of $1000 \mathrm{MC}$ scans for equilibration and 10000 to $50000 \mathrm{MC}$ scans for production.

\section{RESULTS AND DISCUSSION}

Bioenergetics of Transmembrane Transport. In this section, we study the thermodynamics of the transmembrane transport of ammonia and ammonium from an overall perspective without regard to mechanistic details. The free energy for the import of a permeant is given by the difference in the electrochemical potential of the permeant between the inside phase and the outside phase e $^{64,92-94}$

$$
\Delta G^{\text {transfer }}=\bar{\mu}^{\text {in }}-\bar{\mu}^{\text {out }}
$$

The electrochemical potentials of ammonia and ammonium are calculated as described in the Methods section. The electrochemical potential of the permeant species depends on the activity of the permeant species and the electrostatic potential in the respective bulk solvent phase. Ammonia and ammonium are interconvertible through protonation as described by eq 5 . This protonation equilibrium leads to a $\mathrm{pH}$-dependence of the activities of ammonia and ammonium and thus of their electrochemical potentials (Figure S1 of the Supporting Information). Thus, there are three factors that determine the electrochemical potentials of ammonia and ammonium in a given solvent phase. The first factor is the total activity of ammonium and ammonia that is measured by the $\mathrm{pN}$ value defined by eq 11 . The second factor is the relative abundance of the two protonation forms which is determined by the $\mathrm{pH}$ value via $\left(a_{\mathrm{NH}_{3}} / a_{\mathrm{NH}_{4}}{ }^{+}\right)=10^{\mathrm{pH}-\mathrm{p} K_{\mathrm{a}}}$, where $\mathrm{p} K_{\mathrm{a}}=9.25$. The third factor is the electrostatic potential in the respective bulk solvent phase determined by the electric transmembrane potential.

Figure 3 shows plots of the transfer free energy for transport of ammonia or ammonium from the extracellular phase to the intracellular phase. Figure 3a shows plots of the transfer free energy as a function of the intracellular $\mathrm{pH}$ and the protonmotive force (pmf). Figure $3 \mathrm{~b}$ contains the corresponding plots at a fixed intracellular $\mathrm{pH}$ value of 7.0 as functions of the concentration gradient of ammonia/ammonium across the membrane and the pmf. The pmf is the electrochemical potential difference of the proton across the membrane 

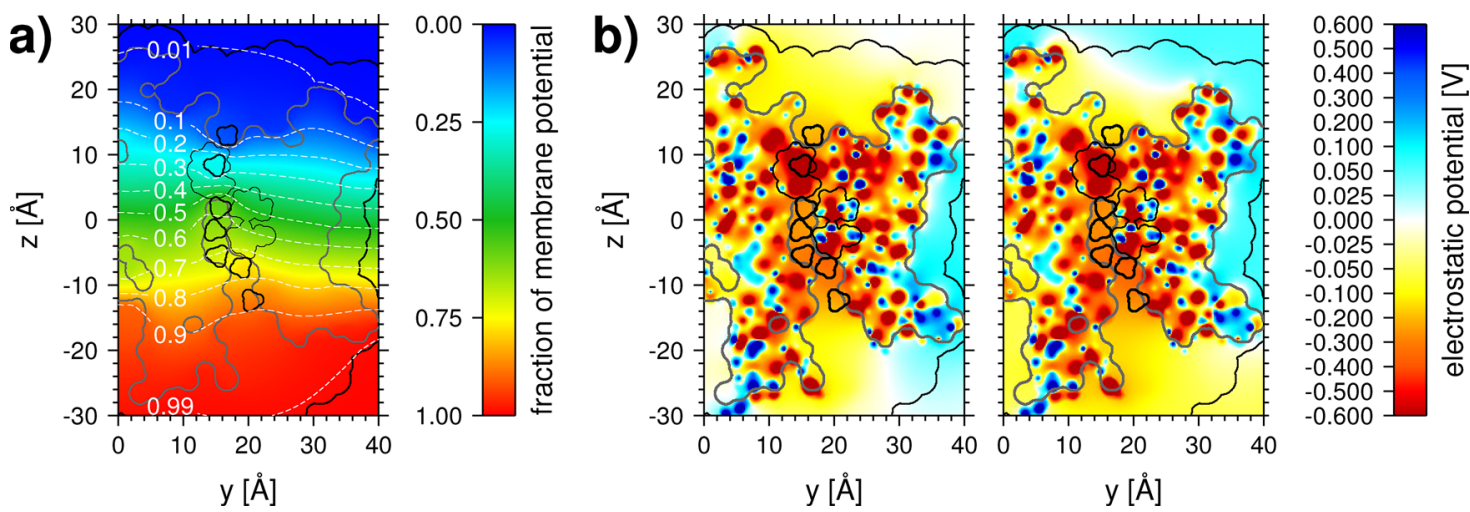

Figure 4. Electrostatic potential distribution in a cross-section through AfAmt-1 as function of the transverse position $\mathrm{z}$ and the lateral position $\mathrm{y}$ in the projection plane. The orientation of the protein is the same as in Figure 2 and the electrostatic potentials are plotted in the same slice plane. (a) The fraction of the electric transmembrane potential (electric distance) as a function of the coordinates y and $\mathrm{z}$. The fraction is color coded (see color bar) and indicated by isocontours. (b) The electrostatic potential under typical conditions $(\mathrm{pH} 7.0$ and $\Delta \psi=-0.12 \mathrm{~V})$. The structure of AfAmt-1 was constructed by setting all sites to their most highly populated instances under the specified conditions. The electrostatic potential is color coded (see color bar). left: electrostatic potential contribution due to the protein charge distribution alone. right: total electrostatic potential due to the protein charge distribution and the electric transmembrane potential.

expressed in terms of a voltage difference acting on the charge of the proton ${ }^{65,92}$

$$
\mathrm{pmf}=-\frac{\ln 10}{\beta F} \Delta \mathrm{pH}+\Delta \psi
$$

where $F$ is the Faraday constant, $\Delta \mathrm{pH}=\mathrm{pH}^{\text {in }}-\mathrm{pH}^{\text {out }}$, and $\Delta \psi$ $=\psi^{\text {in }}-\psi^{\text {out }}$. Here, $\psi$ is the electrostatic potential in the respective bulk solvent phase (far from the membrane). A negative value of the pmf corresponds to the physiological direction of the pmf ( $\psi$ inside negative, outside higher proton activity than inside), while a positive value of the pmf indicates a nonphysiological direction of the pmf. Equation 18 provides a possibility to express the chemical component $\Delta \mathrm{pH}$ and the electrical component $\Delta \psi$ of the proton-motive in a common unit. In this way, we can directly compare the effect of the two pmf components on the transfer free energy. Analogously to $\Delta \mathrm{pH}$, the concentration gradient of the permeants across the membrane is expressed as $\Delta \mathrm{pN}=\mathrm{pN}^{\text {in }}-\mathrm{pN}^{\text {out }}$. A negative value of $\Delta \mathrm{pN}$ indicates that the permeant is less abundant in the outside phase than in the inside phase. This case corresponds to the physiologically relevant situation for microorganisms and plants that have to acquire ammonium as nitrogen source at low ammonium concentrations in the surrounding medium. ${ }^{1}$ Typical ammonium concentrations inside the cell are $\sim 1 \mathrm{mM}$, whereas the ammonium concentration in the surrounding medium is typically significantly lower. ${ }^{1,14,19,23,95,96}$

The left column of Figure 3a shows the effect of the electric transmembrane potential on the transfer free energy. If the electrostatic potential is lower (more negative) in the inside phase than in the outside phase, the import of the positively charged ammonium ion will be thermodynamically favored. In contrast, the import of the uncharged ammonia molecule is unaffected by the electrostatic potential difference between the solvent phases.

The right column of Figure 3a shows the effect of the transmembrane $\mathrm{pH}$ difference on the transfer free energy. If the $\mathrm{pH}$ value of the outside phase is lower than that of the inside phase, the import of $\mathrm{NH}_{4}^{+}$will be thermodynamically favored. This favorable effect of the $\mathrm{pH}$ gradient arises because a fraction of the ammonium ions will be deprotonated in the more alkaline inner phase leading to a free energy gain. However, the magnitude of this energy gain will be very small under physiologically relevant conditions where, on both membrane sides, the $\mathrm{pH}$ value is close to 7.0 and ammonium is the predominant protonation form. In contrast, the import of $\mathrm{NH}_{3}$ is hindered by a transmembrane $\mathrm{pH}$ difference in the physiological direction. The free energy cost arises because a part of the imported ammonia is formed by deprotonation of ammonium at the more acidic extracellular side and reprotonated at the more alkaline intracellular side. Thus, the ammonia uniport is, in this case, thermodynamically equivalent to a net antiport of an ammonium ion and a proton, where the ammonium ion is imported and the proton is exported against the direction of the pmf. $^{4}$

Figure $3 \mathrm{~b}$ shows plots of the transfer free energy for the import of ammonia or ammonium at a fixed intracellular $\mathrm{pH}$ value of 7.0 as a function of the transmembrane concentration gradient of the permeant and the pmf. The permeant concentration gradient $\Delta \mathrm{pN}$ favors the permeant import if the permeant concentration in the outer phase is higher than in the inner phase. Under physiologically relevant conditions for bacteria and plants, however, the concentration of the permeants is lower in the outer phase than in the inner phase. Consequently, the permeant concentration gradient will actually hinder the permeant import by making a positive contribution to the transfer free energy. This unfavorable free energy contribution has to be compensated by coupling the permeant import to the pmf as a driving force. A physiological pmf cannot drive the net import of ammonia as can be seen from the lower row of Figure 3a. However, a physiological pmf can drive the net import of ammonium as can be seen from the upper row of Figure 3a.

In summary, a net uptake of nitrogen at low concentrations of ammonia/ammonium in the extracellular medium is only possible as pmf-driven electrogenic import. A pmf driven transport mode couples the thermodynamically unfavorable import of ammonia to the thermodynamically favorable import of the proton. ${ }^{4,96} \mathrm{~A}$ net electrogenic transport mode can also involve the transport of a part of the substrate as ammonia and another part as ammonium. The import is likely predominantly driven by the electric transmembrane potential since the transfer free energy of ammonium is much more sensitive to 
this component of the pmf than to the $\mathrm{pH}$ gradient (Figure $3 \mathrm{~b})$. Furthermore, $\Delta \psi$ is also the dominant pmf component at the membranes of bacteria ${ }^{1,97}$ and plant root cells. ${ }^{19}$

Electrostatic Potential Across AfAmt-1. In this section, we investigate the possible role of the electrostatic potential in the transport mechanism of AfAmt-1. Figure 4 shows plots of the electrostatic potential distribution across AfAmt-1. Contributions arising from the electric transmembrane potential and the protein charge distribution are shown separately and added up at $\mathrm{pH} 7.0$ and $\Delta \psi=-120 \mathrm{mV}$. Figure 4a shows that the transmembrane potential does not exhibit a simple linear dependence on the coordinate transverse to the membrane. This nontrivial dependence is caused by the complex shape of the molecule and the resulting distribution of the dielectric regions. ${ }^{64}$ A physiological transmembrane potential arises from the separation of positively and negatively charged ions by the membrane, where a net positive charge resides on the extracellular side and a net negative charge of the same magnitude resides on the intracellular side. Thus, the electrostatic transmembrane potential ranges from $-1 / 2 \Delta \psi$ in the bulk extracellular phase to $+1 / 2 \Delta \psi$ in the bulk intracellular phase. The contribution of the electric transmembrane potential to the total electrostatic potential is much lower than the contribution of the protein electrostatic potential for realistic values of $\Delta \psi$.

The protein electrostatic potential is negative in the whole transmembrane pore region (Figure $4 \mathrm{~b}$ ). The electrostatic potential in the vestibule regions and the lumen of the pore can thus aid in the stabilization of a positively charged permeant (see also Figures S2 to S4 of the Supporting Information). The negative electrostatic potential at the extracellular vestibule is also likely to increase the rate of substrate uptake from the extracellular phase by attracting positively charged ammonium ions. The negatively charged side chain of D149 makes the largest contribution to the negative electrostatic potential at the extracellular vestibule. A role of the equivalent residue D160 in EcAmtB in the stabilization of the ammonium ion at the extracellular vestibule has been proposed earlier by Luzhkov et al. $^{36}$

Thermodynamics of Permeant Binding. In this section, we investigate the different factors that influence the thermodynamics of transferring the permeants from the bulk solvent phases to the permeant sites in the transporter pore. The free energy difference associated with this transfer can be formalized as binding free energy of a particular permeant species at a given permeant site in the transporter pore. We defined the unbound state of a permeant site by occupation of its ligand-free binding form. The bound state is defined by occupation of one of the forms of the permeant site with the respective permeant species bound. By comparing the binding free energies for the different permeant species, we can investigate possible implications for the transport mechanism of AfAmt-1.

Figure 5 shows the binding free energies and standard binding free energies at all permeant sites for all permeants considered ( $\mathrm{pH} 7.0, \Delta \psi=0 \mathrm{mV}, \mathrm{pN}$ 3). The standard binding free energy (Figure 5a) extrapolates the binding free energy to a virtual standard concentration of $1.0 \mathrm{~mol} / \mathrm{L}$. The standard binding free energy for the ligand species $x$ can be calculated from the binding free energy for this ligand by removing the explicit dependence of the binding free energy on the activity of the ligand
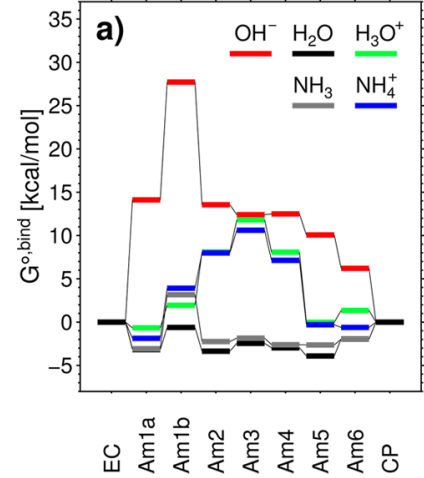

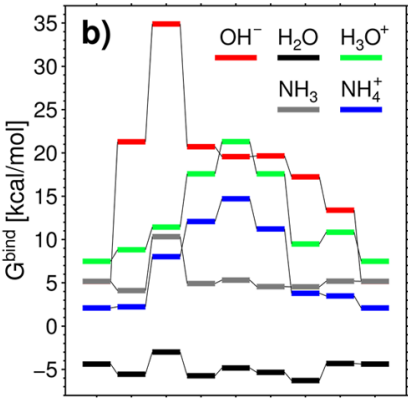

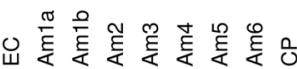

Figure 5. Standard binding free energy (a) and binding free energy (b) for different permeants at the transmembrane pore sites. The $\mathrm{pH}$ is set to 7.0 on both membrane sides. No pmf is applied. The $\mathrm{pN}$ is set to 3.0 on both membrane sides. Connecting lines between the free energy levels are just a guide to the eye and do not imply transition state energies.

$$
G_{x}^{o, \text { bind }}=G_{x}^{\text {bind }}-\beta^{-1} \ln a_{x}
$$

In general, there is also an implicit dependence of the standard binding free energy on the ligand activity if there are other, interacting binding sites for the same ligand. ${ }^{98-100}$ However, since the permeant sites are almost always occupied by an uncharged water molecule under physiologically relevant conditions, the interactions between the permeant sites are limited. Consequently, the implicit dependence of the standard binding free energy on the ligand activity is small in our case.

The standard binding free energy is directly comparable to potential of mean force profiles which are often used in MD studies to analyze the thermodynamics of transmembrane transport. Potential of mean force profiles for the permeation of ammonia and ammonium along the transmembrane pore of homologues of AfAmt-1 obtained from MD studies have been reported. ${ }^{32,39,48-50}$ We compared the potential of mean force at the positions equivalent to our permeant sites to our standard binding free energies. The potential of mean force profiles for the permeation of $\mathrm{NH}_{3}$ through $\mathrm{EcAmtB} \mathrm{B}^{32,39,48}$ and $\mathrm{NeRh} 50^{50}$ are comparable to the profile of our standard binding free energies. The corresponding profiles for the permeation of $\mathrm{NH}_{4}^{+}$obtained from the MD studies show a significantly less favorable thermodynamics of permeation than our standard binding free energies. This discrepancy arises most likely from the presence of water in the pore lumen in our simulation and its absence in the molecular dynamics studies. In their recent work, employing a polarizable force field, Lamoureux et al. found even more favorable standard binding free energies for ammonium in the hydrophobic pore lumen than found here. ${ }^{49}$ This finding is based on the explicitly included polarization effect whose predicted magnitude is, however, surprisingly large.

Upon transfer from the bulk solvent into the transmembrane pore, the permeant is partially desolvated. This desolvation leads to a positive contribution to the binding free energy, often termed desolvation penalty, which disfavors binding. This effect is especially pronounced for charged permeant species. Water present in the pore lumen can partially compensate for the lost hydration hull and thus reduce the free energy cost for desolvation of the charged permeant. The desolvation penalty in our simulations is alleviated in this way because the surrounding permeant sites are almost always occupied by 

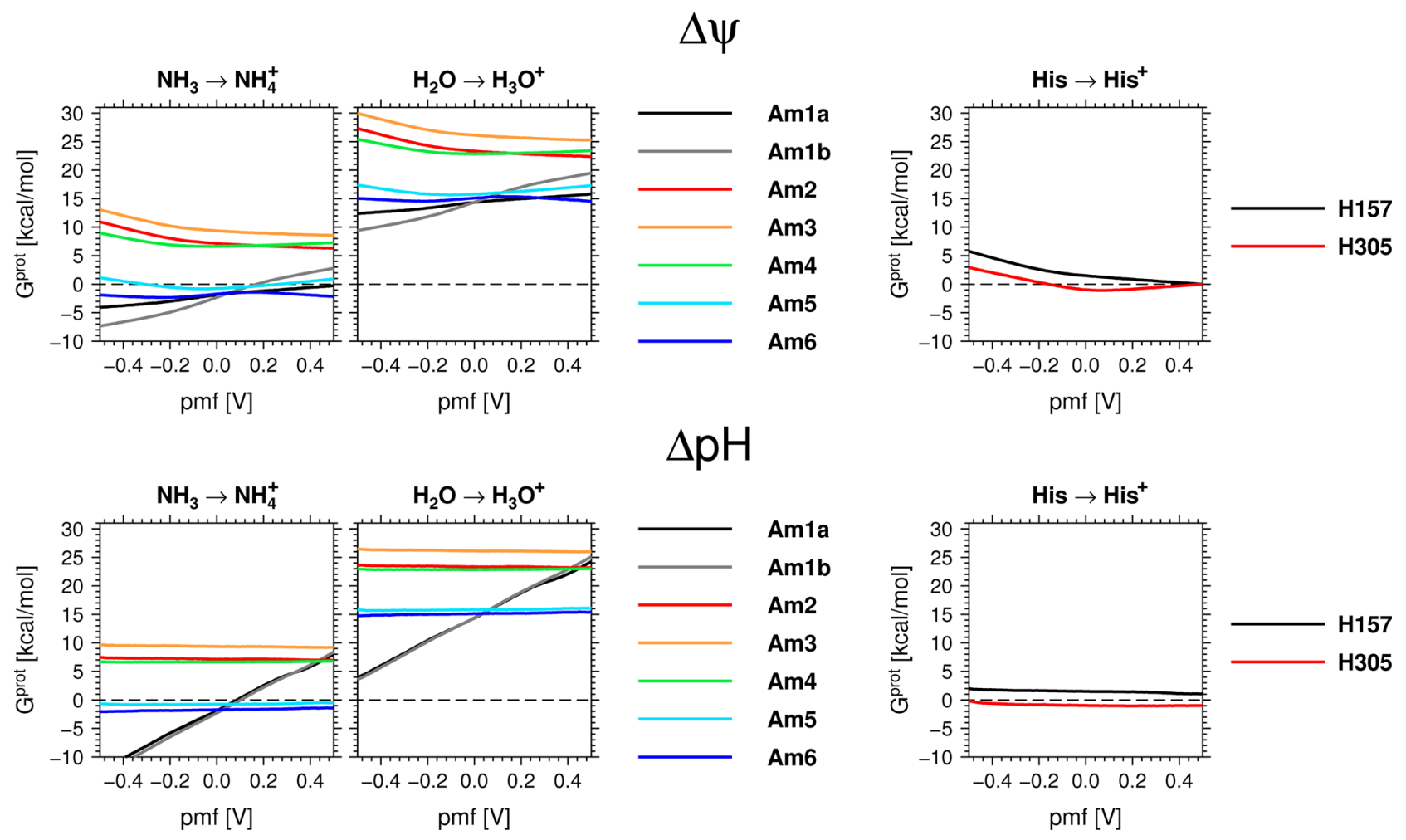

Figure 6. Protonation free energy for the twin-His motif and the permeants ammonia and water at the transmembrane pore sites as functions of a proton-motive force. Upper block: pmf consists entirely of an electric transmembrane potential. The $\mathrm{pH}$ value is set to 7.0 on both membrane sides. Lower block: pmf consists entirely of a transmembrane $\mathrm{pH}$ difference. The intracellular $\mathrm{pH}$ value is set to 7.0 . The extracellular $\mathrm{pH}$ value is given by $7.0+\mathrm{pmf} / 59 \mathrm{mV}$. For both blocks, the $\mathrm{pN}$ is set to 3.0 on both membrane sides.

water. Note that the possibility of empty binding sites is included in our model, i.e., the water molecules are free to leave the transporter pore. The question whether or not the pore lumen is occupied by water is thus a very important point in determining the electrostatic barrier for permeation of ammonium through the pore. The importance of water for the stabilization of the protonated $\mathrm{NH}_{4}^{+}$ion relative to the deprotonated $\mathrm{NH}_{3}$ molecule has already been pointed out by Bostick and Brooks ${ }^{32,33}$ and Bernèche et al. ${ }^{5,46}$ Although intraluminal water is not observed in most of the MD simulations reporting the potential of mean force profiles, other MD studies show stable ${ }^{46,49}$ or transient ${ }^{36,37}$ hydration of the pore lumen. Most notably, a recent $\mathrm{MD}$ simulation of EcAmtB with a polarizable force field also showed stable hydration of the pore. ${ }^{49}$ Thus, the protein-water interactions might be influenced by the chosen water model. ${ }^{101,102}$ Along these lines, a recent work studied the role of the water model in binding free energy calculations and found that a more detailed 5-site water model like the one used here yielded more reliable predictions than older, three-site water models like those used in the MD studies that did not show hydration of the hydrophobic pore lumen of EcAmtB. ${ }^{103}$

The standard binding free energy directly measures the difference between the interactions of the permeant with the protein environment and the bulk solvent. Thus, $G^{\circ \text {,bind }}$ can be used to analyze whether AfAmt-1 has an intrinsic selectivity for a certain permeant species over other permeant species. The standard binding free energies reveal that AfAmt-1 is selective for $\mathrm{NH}_{4}^{+}$over $\mathrm{H}_{3} \mathrm{O}^{+}$at the vestibule regions (Amla, $\mathrm{Amlb}$, and Am7) and at the center of the pore lumen (Am3 and Am4). This selectivity is based on the higher desolvation penalty for the smaller and more polar $\mathrm{H}_{3} \mathrm{O}^{+}$ion. In contrast, a selectivity of AfAmt-1 for $\mathrm{NH}_{3}$ over $\mathrm{H}_{2} \mathrm{O}$ is not apparent from our results, which is perhaps not surprising given the similar physicochemical properties of these molecules. Such a selectivity might, however, also arise from differences in the free energy barriers between the permeant positions which are not included in our calculations. Binding of the $\mathrm{OH}^{-}$ion is very unfavorable because of the high desolvation penalty and the unfavorable electrostatic interaction with the protein (Figure $4 b$ ).

The binding free energy extends the standard binding free energy by adding the effect of the actual activities of the different permeant species (Figure $5 b$ ). The binding free energy is more favorable if the permeant is more abundant in the respective solvent phase and less favorable if the permeant is less abundant. The activity of the different protonation forms of ammonia and water is determined by the $\mathrm{pH}$ value of the respective solvent phase (see Methods section). At neutral $\mathrm{pH}$ values, $\mathrm{NH}_{4}{ }^{+}$is much more abundant than $\mathrm{NH}_{3}$. Similarly, $\mathrm{H}_{2} \mathrm{O}$ is the predominant protonation form of water, whereas $\mathrm{H}_{3} \mathrm{O}^{+}$ and $\mathrm{OH}^{-}$are very rare. Thus, the contribution of the permeant activity to the binding free energy strongly favors the binding of $\mathrm{NH}_{4}^{+}$over that of $\mathrm{NH}_{3}$ and the binding of $\mathrm{H}_{2} \mathrm{O}$ over that of $\mathrm{H}_{3} \mathrm{O}^{+}$and $\mathrm{OH}^{-}$. In addition, the total activity of the water species will normally be much higher than that of the ammonia species (typically $c_{\mathrm{NH}_{3}}+c_{\mathrm{NH}_{4}}{ }^{+}<10^{-3} \mathrm{~mol} / \mathrm{L}$ while $c_{\mathrm{H}_{2} \mathrm{O}} \approx$ $55.5 \mathrm{~mol} / \mathrm{L}$ ). Due to its high abundance, the binding of water is much more favorable than the binding of any other permeant species at all permeant sites. Consequently, the permeant sites are almost always occupied by water and only occasionally by the other permeant species. Thus, it seems unlikely that the transport mechanism of AfAmt-1 involves the binding of more 

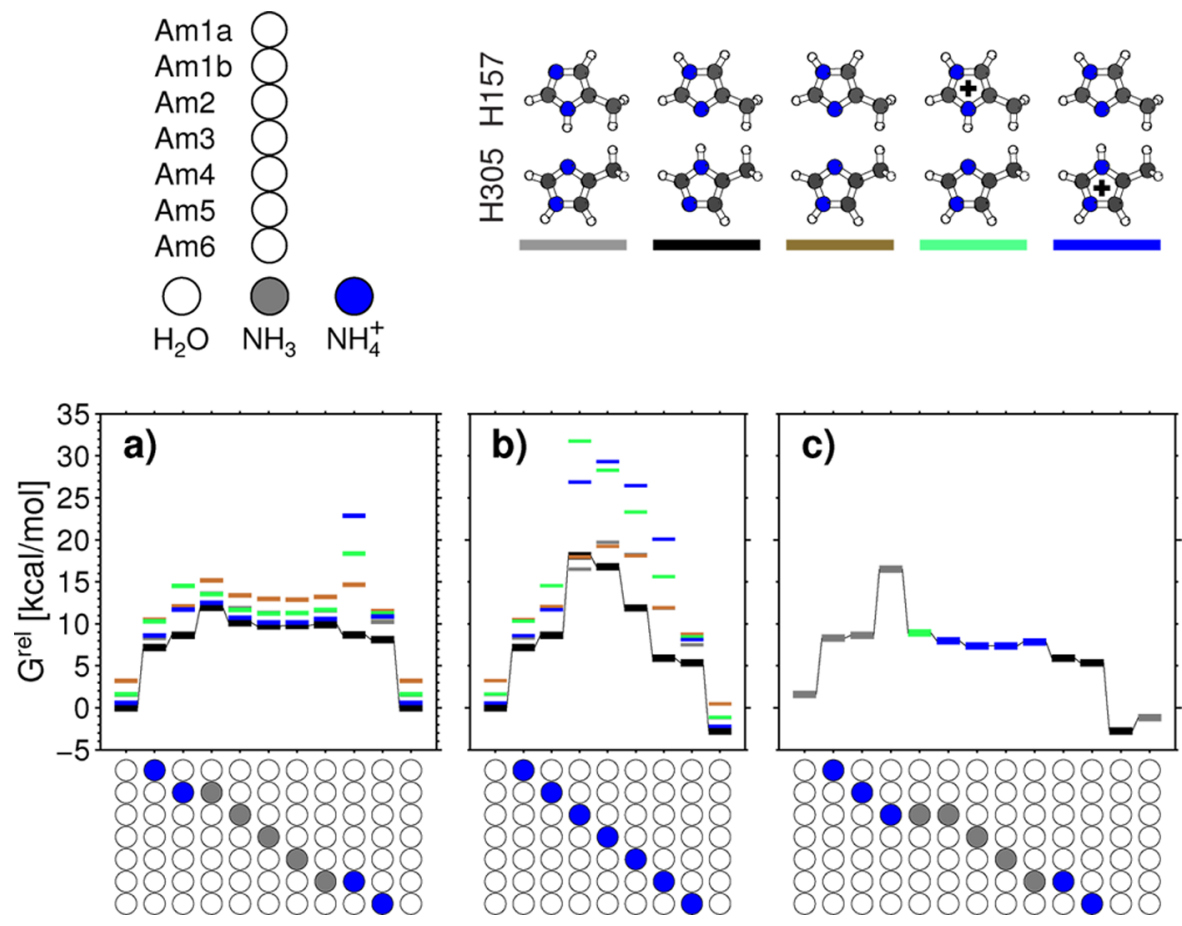

Figure 7. Free energy level scheme for states of the permeant sites and the twin-His motif occurring during different proposals for the permeation mechanism. The species present at the permeant sites (Amla to Am6) are indicated at the horizontal axis (gray, $\mathrm{NH}_{3} ; \mathrm{blue} \mathrm{NH}_{4}^{+} ; \mathrm{white}_{2} \mathrm{H}_{2} \mathrm{O}$ ). The free energy of each state is plotted relative to the free energy of the state with all permeant sites occupied by water, H157 adopting the N $\epsilon$ protonated tautomer and $\mathrm{H} 305$ adopting the $\mathrm{N} \delta$-protonated tautomer. The energy levels are color coded according to the configuration adopted by the twin-His motif as defined by the legend. Connecting lines between the free energy levels are just a guide to the eye and do not imply transition state energies. The $\mathrm{pH}$ is set to 7.0 on both membrane sides. The $\mathrm{pN}$ is set to 3.0 on both membrane sides. $\Delta \psi$ is set to $-0.12 \mathrm{~V}$. (a) Electroneutral $\mathrm{NH}_{3}$ uniport; (b) electrogenic $\mathrm{NH}_{4}^{+}$uniport not involving protonation state changes of the twin-His motif; (c) electrogenic $\mathrm{NH}_{3} / \mathrm{H}^{+}$symport involving protonation state changes of the twin-His motif. It is not clear how the restoration of the twin-His motifs configuration between the last two stages of this mechanistic scheme will occur microscopically. The restoration might involve a transient reprotonation of the twin-His motif, proton transfer along the water molecules in the pore lumen and/or a transient reorientation of the histidine side chains.

than one molecule of the permeants ammonia or ammonium per transporter monomer at the same time. Crystallographic studies showed residual electron density in the pore lumen of EcAmtB. ${ }^{16,17,34,35}$ As noted earlier by Zheng et al. ${ }^{17}$ and ourselves, ${ }^{18}$ it is impossible to reliably distinguish between water and ammonia as occupants of the permeant sites on the basis of the experimentally determined electron density. Bernèche and co-workers pointed out the importance of the high water concentration for the question whether the permeant sites are occupied by ammonium/ammonia or water. 5,17 These considerations and the findings from our calculations argue for water as predominant occupant of the permeant sites at least for AfAmt-1 and possibly also for its structurally highly similar homologues. Plots of the binding free energies for all permeant species at all permeant sites as a function of the pmf components can be found in section $F$ of the Supporting Information.

Protonation Free Energies of the Permeant Sites and the twin-His Motif. In this section, we study the protonation free energies of ammonia and water at the permeant sites and of the twin-His residues. Figure 6 shows plots of these protonation free energies as functions of an electric transmembrane potential or a transmembrane $\mathrm{pH}$ gradient. The settings chosen in the calculation of the protonation free energies are the same as those used in the calculation of the permeant binding free energies above. The protonation free energies of the twin-His residues and of water and ammonia at the intracellular permeant sites (Am2 to $\mathrm{Am} 7)$ are only weakly dependent on the $\mathrm{pH}$ gradient because the intracellular $\mathrm{pH}$ value is constant. The protonation free energies of $\mathrm{NH}_{3}$ and $\mathrm{H}_{2} \mathrm{O}$ at the extracellular vestibule (Amla and Amlb) are roughly linearly dependent on the $\mathrm{pH}$ gradient. This dependence stems from the dependence of the extracellular $\mathrm{pH}$ value, and thus of the extracellular proton activity, on the $\mathrm{pH}$ gradient.

The effect of the electric transmembrane potential on the protonation free energies arises from its influence on the intrinsic energies of the binding sites and on the proton electrochemical potentials of the solvent phases. The transmembrane potential favors the release of protons to the solvent phase with the more negative potential and the uptake of protons from the solvent phase with the more positive potential. This is why the transmembrane potential has an qualitatively opposite effect on the protonation free energy of intracellular and extracellular binding sites. The effect of the transmembrane potential increases with the distance of the binding site from the respective outer surface of the protein (Figure 4a). This dependence can be understood from the difference in the electrostatic potential between the respective bulk solvent phase and the position of the binding site. A larger electrostatic potential difference will lead to a larger magnitude of the energy that is gained or spent in transferring the proton from the bulk solvent to the binding site.

The protonation free energy of water is less favorable than the protonation free energy of ammonia at all permeant sites. This difference is mainly caused by the lower intrinsic proton affinity of water relative to ammonia. An additional 
contribution arises from the higher desolvation penalty for the smaller and more polar hydronium ion relative to the ammonium ion. The protonation free energy of the permeants at the vestibular permeant sites (Amla, Amlb, Am6, and Am7) is much more favorable than at the luminal permeant sites (Am2, Am3, and Am4). The protonation free energy of water at the luminal permeant sites is so unfavorable that it seems unlikely that a hydronium ion at the luminal sites is involved in the conduction mechanism. In contrast, the lower protonation free energies of ammonia let it seem possible that an ammonium ion can be transiently stabilized at the intraluminal permeant sites. However, the protonation free energies indicate that the permanent protonation of ammonia is only favorable at the vestibular permeant sites but not at the intraluminal permeant sites. This finding is consistent with the findings of previous theoretical studies on $\mathrm{EcAmtB}^{32,33,36-48}$ and NeRh $50^{50}$ and the binding of xenon to the intraluminal permeant sites of xenon-pressurized AfAmt-1 crystals. ${ }^{18}$

The twin-His residues are the only titratable sites of the protein whose protonation probability is strongly dependent on the binding of ammonia/ammonium to the permeant sites (see plots in section $\mathrm{E}$ of the Supporting Information). The possibilities of an active role of the twin-His motif as transient proton acceptor for the substrate ammonium ${ }^{5,17,34,35}$ or a passive role as hydrogen bond donor to the permeants passing the pore lumen have been noted earlier. ${ }^{16}$ The protonation free energies of the twin-His residues have small magnitudes over the whole range of conditions studied. The small free energy differences between the protonated and deprotonated forms of these residues indicate that transient changes between the protonation states are easily possible. Thus, the twin-His motif could play an active role in a possible electrogenic conduction mechanism of AfAmt-1. This possibility is investigated further in the next section.

Thermodynamics of Possible Permeation Mechanisms. For investigating the thermodynamics of possible permeation mechanisms, we concentrate on the permeant sites and the two histidine residues forming the twin His-motif. We considered microscopic states for these sites, while all other sites can freely equilibrate. For the twin-His motif, we considered all five thermodynamically viable permutations of the histidine protonation forms as depicted in the legend of Figure 7. We computed the free energy of all states with one or two permeants other than water relative to the fully water occupied state with the thermodynamically most favorable protonation form of the twin His motif (H157 singly protonated at the $\mathrm{N} \epsilon$ atom and $\mathrm{H} 305$ singly protonated at the $\mathrm{N} \delta$ atom). The full sets of computed relative free energies of the states are depicted in Figures S26 to S29 of the Supporting Information. Occupation of more than two sites at the same time by other permeant species than water is thermodynamically very unfavorable and thus unlikely to be involved in the permeation mechanism. Figure 7 shows free energy profiles for the possible permeation mechanisms considered herein. We did not calculate free energy barriers between the intermediate states of the permeation mechanism. Potential of mean force profiles for the permeation of $\mathrm{NH}_{3}$ and $\mathrm{NH}_{4}{ }^{+}$through EcAmtB are available from MD studies. ${ }^{32,33,48,49}$ These profiles indicate that the barriers for permeant translocation between the permeant sites are small relative to the overall electrostatic barrier for the permeation of the pore lumen by the ammonium ion. Thus, we believe that the inclusion of these barriers is of minor importance for evaluating the thermodynamic viability of the transport mechanisms.

The parameters in the calculations were set to standard values that could be encountered at the membrane of microorganisms. The $\mathrm{pH}$ value was set to 7.0 on both membrane sides. The electric transmembrane potential was set to $-0.12 \mathrm{~V}$. A transmembrane $\mathrm{pH}$ gradient was not applied because its effect on the transport and binding thermodynamics is much smaller than that of the electric transmembrane potential (see above). Typically, $\Delta \psi$ is also the dominant component of the pmf. ${ }^{1,19,97}$ The $\mathrm{pN}$ value was set to 3.0 on both membrane sides. Figure 7 shows the free energy profiles of possible conduction mechanisms. Taking into account the permeant concentrations and the electrochemical transmembrane gradients has a significant effect on the free energy profiles. For comparison, Figure S30 of the Supporting Information shows the same free energy profiles at standard total concentration of the permeants and at zero electrochemical transmembrane gradients.

Figure $7 \mathrm{a}$ shows the electroneutral $\mathrm{NH}_{3}$ uniport mechanism first proposed in microscopic detail by Khademi et al. on the basis of the hydrophobicity of the transmembrane pore. ${ }^{16}$ This mechanism was supported by previous theoretical studies on $E_{c A m t B}{ }^{32,33,36-48}$ and NeRh50 ${ }^{50}$ which concentrated on the ammonia or ammonium uniport mechanisms. The $\mathrm{NH}_{3}$ uniport mechanism does not involve protonation state changes of the twin-His motif. Ammonium is recruited at Amla and deprotonated somewhere close to Amlb. The proton is left behind in the extracellular phase. The neutral ammonia molecule permeates the hydrophobic pore lumen consisting of $\mathrm{Am} 2, \mathrm{Am} 3$ and $\mathrm{Am} 4$, and is reprotonated with a proton from the intracellular phase at Am5. Finally, the ammonium ion is released to the intracellular phase. The reasoning for the deprotonation of $\mathrm{NH}_{4}^{+}$at $\mathrm{Am} \mathrm{lb}$ and its reprotonation at $\mathrm{Am} 5$ is based on the high protonation free energy of ammonia at the intervening intraluminal permeant sites.

Figure $7 \mathrm{~b}$ shows an electrogenic $\mathrm{NH}_{4}^{+}$uniport mechanism that does not involve protonation state changes of the twin-His motif (net transport of $\mathrm{NH}_{4}^{+}$). The ammonium ion is recruited from the extracellular phase at Amla, permeates the whole transmembrane pore passing the intermediate permeant sites and is released from Am7 to the intracellular phase. The permeation of the transmembrane pore is associated with an electrostatic energy barrier of ca. $15 \mathrm{kcal} / \mathrm{mol}$.

Figure $7 \mathrm{c}$ shows a possible electrogenic $\mathrm{NH}_{3} / \mathrm{H}^{+}$symport mechanism (net transport of $\mathrm{NH}_{4}^{+}$). The most important features of this mechanism have been proposed by Bernèche and co-workers. ${ }^{5}$ Ammonium is recruited from the extracellular phase at Amla, loses its hydration shell at Amlb and is transferred to Am2. At Am2, the ammonium ion is deprotonated by $\mathrm{H} 157$. An escape of the $\mathrm{NH}_{3}$ molecule back to the intracellular vestibule might be hindered by the preferential permeability of the Phe gate for $\mathrm{NH}_{4}{ }^{+}$relative to $\mathrm{NH}_{3}$ reported by Bernèche and co-workers. ${ }^{5}$ The protonation of the twin-His motif is stabilized by the hydrogen bond between the two central $\mathrm{N} \delta$ nitrogen atoms. This hydrogen bond also enables the twin-His motif to easily change between the two protonated configurations (green and blue in Figure 7) via proton transfer between the two central $\mathrm{N} \delta$ nitrogen atoms. In this way, the proton is transferred from $\mathrm{H} 157$ to $\mathrm{H} 305$ at the intracellular side of the pore. The neutral ammonia molecule permeates the hydrophobic pore lumen and is reprotonated by $\mathrm{H} 305$ at Am5. Finally, the ammonium ion is transferred to Am6 
and released to the intracellular phase. This mechanism has a slightly lower electrostatic barrier for the permeation of the pore lumen than the $\mathrm{NH}_{4}{ }^{+}$uniport mechanism (ca. $12 \mathrm{kcal} /$ $\mathrm{mol}$ ). As noted by Bernèche et al., ${ }^{5}$ this mechanism poses the problem of how to reset the tautomeric forms of the twin-His residues to those occupied initially. The reset could involve a transient reprotonation of the twin-His motif from the intracellular phase. In addition, the restoration might involve proton transfer along the water molecules in the pore lumen in a Grotthuss mechanism and/or a transient reorientation of the histidine side chains. The uncertainty about the precise mechanism of the reset makes it impossible at present to quantify the size of the associated free energy barrier with confidence. Based on gas-phase QM calculations, Lamoureux et al. estimate the free energy barrier for this reset to be no larger than $15 \mathrm{kcal} / \mathrm{mol}$ if the reset involves water molecules in the pore and a transiently formed $\mathrm{OH}^{-}{ }^{49}$ As discussed above, the formation of $\mathrm{a} \mathrm{OH}^{-}$ion is thermodynamically very unfavorable within the protein environment (see Thermodynamics of Permeant Binding section). Within our model, the barrier for the transient formation of the $\mathrm{OH}^{-}$ion would be about 22 $\mathrm{kcal} / \mathrm{mol}$ (Figures S26 and S28 of the Supporting Information). This high free energy barrier might, however, be lowered if polarization and quantum effects are explicitly included. A reset mechanism that involves reorientation of the twin-His side chains is found by Lamoureux et al. to be unlikely because the associated barrier is larger than $20 \mathrm{kcal} / \mathrm{mol}$.

Which transport mechanism is now likely to be operative? The low overall free energy barrier of $12 \mathrm{kcal} / \mathrm{mol}$ associated with the electroneutral $\mathrm{NH}_{3}$ uniport mechanism indicates that the mechanism is kinetically feasible. An important counter argument against an $\mathrm{NH}_{3}$ uniport as exclusively operative mechanism is the lack of a net thermodynamic driving force under physiologically relevant conditions (see Bioenergetics of Transmembrane Transport section). In contrast, an $\mathrm{NH}_{3}$ uniport is even hindered if a physiological $\mathrm{pH}$ gradient is applied in addition to the electric transmembrane potential (Figure 3); that is, ammonia would be exported instead of imported. With a $\mathrm{pH}$ gradient of $\Delta \mathrm{pH}=1 \mathrm{pH}$ unit, the ammonia import would be hindered by $1.3 \mathrm{kcal} / \mathrm{mol}$. In contrast, an electrogenic transport mechanism involving $\mathrm{NH}_{4}{ }^{+}$ can be driven by the electric transmembrane potential (Figure 3).

Amt/Rh proteins of microorganisms, plants and mycorrhizal fungi seem to be expressed only if the availability of nitrogen, sources is low, i.e., if the ammonium concentration in the medium is low.,104-107 This finding underlines the necessity for these life forms to drive the ammonium import by the electric transmembrane potential as pointed out earlier by ourselves and other authors. ${ }^{1,4,14,18}$ The free energy schemes for the electrogenic mechanisms indicate a sizable total free energy barrier of $15-18 \mathrm{kcal} / \mathrm{mol}$ for the permeation of the transporter pore. The main contribution to this free energy barrier is the cost of the electrostatic desolvation of the ammonium ion upon transfer from the aqueous solvent to the hydrophobic pore lumen $(12-15 \mathrm{kcal} / \mathrm{mol})$. The desolvation penalty might be lowered further when explicitly accounting for electronic polarization, which is especially significant in the interaction of aromatic residues with $\mathrm{NH}_{4}{ }^{+} \cdot{ }^{49,75}$ The electrogenic mechanisms seem to be thermodynamically feasible especially when considering the high optimal growth temperature of $A$. fulgidus of ca. $80{ }^{\circ} \mathrm{C} .{ }^{108,109}$ The similar magnitude of the free energy barriers for the electrogenic mechanism considered and the uncertainty about the free energy barrier associated with the last step of the $\mathrm{NH}_{3} / \mathrm{H}^{+}$symport mechanism do not allow us to prefer one of the mechanism on the basis of the free energy profiles.

Although our calculations concentrated on AfAmt-1 and did not include other homologues, we do believe that many arguments are transferable to other homologues. This belief is based on the high structural similarity of Amt and Rh proteins revealed by the available crystal structures. ${ }^{13,16-18,35,110,111}$ An electrogenic transport was reported on the basis of experiments on plant ${ }^{19-24}$ and microbial $\mathrm{Amts}^{25-27}$ and on human $\mathrm{Rh}$ proteins ${ }^{30}$ implying the involvement of $\mathrm{NH}_{4}^{+}$as substrate. In seeming contradiction, an involvement of $\mathrm{NH}_{3}$ as substrate was implied by experiments reported for EcAmtB ${ }^{16}$ and some Rh proteins. ${ }^{28,29,112,113}$ These experiments indicated an intracellular alkalinization accompanying the transport which was interpreted to originate from reprotonation of the transported $\mathrm{NH}_{3}$ in the intracellular phase. ${ }^{16}$ The significance of these findings for the mechanism of $\mathrm{Amt} / \mathrm{Rh}$ proteins is, however, debated. $^{4,31}$ As pointed out earlier by Musa-Aziz et al., an alkalinization of the intracellular phase occurs already if a fraction of the overall imported ammonia/ammonium is transported as neutral ammonia. ${ }^{11,114}$ In addition, the experiments showed that the transport was stimulated by slight increases in the extracellular $\mathrm{pH}$ value while keeping a $\mathrm{pH}$ value well below the $\mathrm{p} K_{\mathrm{a}}$ of ammonium. Under these conditions, a $\mathrm{pH}$ increase will only have a significant effect on the abundance and availability of $\mathrm{NH}_{3}$ while the abundance of $\mathrm{NH}_{4}{ }^{+}$is merely affected. Bakouh et al. observed sensitivity of the transport through human RhCG to the transmembrane potential and to the extracellular $\mathrm{pH}$ value. ${ }^{30}$ These finding lead them to propose that $\mathrm{NH}_{3}$ and $\mathrm{NH}_{4}^{+}$are directly involved in the transport mechanism. The function of molecular systems is inherently stochastic. Thus, the mechanistic schemes shown in Figure 7 may be seen as limiting cases. The electroneutral $\mathrm{NH}_{3}$ uniport may also contribute to the total permeant flux across the membrane as long as a parallel electrogenic transport supplies an overall thermodynamic driving force.

The prominent role attributed to the twin-his motif in the proposed $\mathrm{NH}_{3} / \mathrm{H}^{+}$symport mechanism is consistent with the high degree of its conservation among $\mathrm{Amt} / \mathrm{Rh}$ proteins. ${ }^{2,5,34,115}$ The histidine residues can in some cases be substituted by glutamate or aspartate ${ }^{34,115}$ which might be able to perform the same function. ${ }^{49}$ There are, however, also some rare cases as in some $\mathrm{Rh}$ proteins where no equivalent of the twin-His motif is present. ${ }^{12}$

From our calculations, it can not be excluded that different transport mechanisms are operative in different Amt/Rh proteins as advocated by some workers. ${ }^{9,10,116,117}$ It is, however, also conceivable that the Amt/Rh proteins can support different transport modes. The transport mechanism actually operational would then depend on the driving force available in a particular system. More work is required to gain certainty in this respect.

\section{CONCLUSIONS AND OUTLOOK}

We investigated the thermodynamics of different possible transport mechanisms of AfAmt-1. Both, electrogenic and electroneutral permeation mechanisms were found to be thermodynamically viable. A net transport of substrate across the membrane requires the presence of a thermodynamic driving force. ${ }^{64,92,93}$ Microorganisms like A. fulgidus and plants have to acquire ammonium at low external ammonium concentrations. $14,19,23,95,96$ We found that, in this case, the 
driving force for the substrate import can only be provided by the electric transmembrane potential implying an electrogenic transport mode. We computed free energy profiles for different electrogenic transport mechanisms and found that $\mathrm{NH}_{4}^{+}$ uniport and $\mathrm{NH}_{3} / \mathrm{H}^{+}$symport are thermodynamically viable. Important factors that allow the permeation of the pore lumen by ammonium are the negative electrostatic potential of the protein and water present in the pore lumen. These factors alleviate the free energy cost of desolvating the ammonium ion upon transfer from the solvent to the hydrophobic pore lumen. The possible $\mathrm{NH}_{3} / \mathrm{H}^{+}$symport mechanism involves the genetically conserved twin-His motif as intermediate acceptor of the cotransported proton. Further experimental and theoretical work is needed to discern between the different possible electrogenic mechanisms and to obtain a deeper understanding of their microscopic details. In particular, it would be interesting to investigate the stoichiometry of protons and ammonia transported as functions of the physical conditions such as $\mathrm{pH}$ value, electric transmembrane potential, and transmembrane $\mathrm{pH}$ gradient. Theoretical methods that provide time information could be used to study microscopic details of the transport kinetics. Extensions of our microstate description to model the kinetics of molecular systems have already been applied successfully and might be used fruitfully in future studies of AfAmt-1. ${ }^{58,118-121}$

The formalism developed herein for the treatment of ligands with multiple binding forms is generally applicable. Often, the ligand itself can occur in multiple protonation forms which leads to a coupling of the protonation equilibria of both binding partners and the receptor-ligand binding equilibrium. The coupling of protein-ligand binding equilibria to the protonation equilibria of the binding partners occurring in such cases is currently receiving increasing attention. ${ }^{5,122-134}$ Our approach is thus also interesting within a wider scope of binding free energy calculations in general, for example in combination with a constant $\mathrm{pH} \lambda$-dynamics method. ${ }^{135-137}$

\section{ASSOCIATED CONTENT}

\section{S Supporting Information}

Parameters used in the simulations, derivations of expressions for the chemical potentials and the transfer free energies of the investigated permeants, and addititional figures referenced in the text. This material is available free of charge via the Internet at http://pubs.acs.org.

\section{AUTHOR INFORMATION}

\section{Corresponding Author}

*E-mail: thomas.ullmann@uni-bayreuth.de; andrade@bio. chemie.uni-freiburg.de; matthias.ullmann@uni-bayreuth.de.

\section{Notes}

The authors declare no competing financial interest.

\section{REFERENCES}

(1) Kleiner, D. FEMS Microbiol. Lett. 1985, 32, 87-100.

(2) Howitt, S. M.; Udvardi, M. K. BBA-Biomembr. 2000, 1465, 152170.

(3) Wirén, N.; Merrick, M. Regulation and function of ammonium carriers in bacteria, fungi, and plants. In Molecular mechanisms controlling transmembrane transport; Springer: Berlin, 2004; Vol. 9, pp 95-120.

(4) Andrade, S. L. A.; Einsle, O. Mol. Membr. Biol. 2007, 24, 357365 .
(5) Lamoureux, G.; Javelle, A.; Baday, S.; Wang, S.; Bernèche, S. Transfus. Clin. Biol. 2010, 17, 168-175.

(6) Khademi, S.; Stroud, R. M. Physiol. 2006, 21, 419-429.

(7) Khademi, S.; Stroud, R. M. Gas channels for ammonia. In Structural biology of membrane proteins; The Royal Society of Chemistry: London, 2006; pp 212-234.

(8) Winkler, F. Pflugers Arch. Eur. J. Phy. 2006, 451, 701-707.

(9) Ludewig, U.; Neuhäuser, B.; Dynowski, M. FEBS Lett. 2007, 581, 2301-2308.

(10) Ludewig, U.; von Wirén, N.; Rentsch, D.; Frommer, W. Genome Biol. 2001, 2, reviews1010.1-reviews 1010.5 .

(11) Boron, W. F. Exp. Physiol. 2010, 95, 1107-1130.

(12) Conroy, M. J.; Bullough, P. A.; Merrick, M.; Avent, N. D. Br. J. Hamaetol. 2005, 131, 543-551.

(13) Gruswitz, F.; Chaudhary, S.; Ho, J. D.; Schlessinger, A.; Pezeshki, B.; Ho, C.-M.; Sali, A.; Westhoff, C. M.; Stroud, R. M. Proc. Natl. Acad. Sci. U.S.A. 2010, 107, 9638-9643.

(14) Boogerd, F. C.; Ma, H.; Bruggeman, F. J.; van Heeswijk, W. C.; García-Contreras, R.; Molenaar, D.; Krab, K.; Westerhoff, H. V. FEBS Lett. 2011, 585, 23-28.

(15) Soupene, E.; He, L.; Yan, D.; Kustu, S. Proc. Natl. Acad. Sci. U.S.A. 1998, 95, 7030-7034.

(16) Khademi, S.; O’Connell, J.; Remis, J.; Robles-Colmenares, Y.; Miercke, L. J. W.; Stroud, R. M. Science 2004, 305, 1587-1594.

(17) Zheng, L.; Kostrewa, D.; Bernèche, S.; Winkler, F. K.; Li, X.-D. Proc. Natl. Acad. Sci. U.S.A. 2004, 101, 17090-17095.

(18) Andrade, S. L. A.; Dickmanns, A.; Ficner, R.; Einsle, O. Proc. Natl. Acad. Sci. U.S.A. 2005, 102, 14994-14999.

(19) Wang, M. Y.; Glass, A. D. M.; Shaff, J. E.; Kochian, L. V. Plant Physiol. 1994, 104, 899-906.

(20) Ludewig, U.; von Wirén, N.; Frommer, W. B. J. Biol. Chem. 2002, 277, 13548-13555.

(21) Mayer, M.; Dynowski, M.; Ludewig, U. Biochem. J. 2006, 396, 431-437.

(22) Mayer, M.; Schaaf, G.; Mouro, I.; Lopez, C.; Colin, Y.; Neumann, P.; Cartron, J.-P.; Ludewig, U. J. Gen. Physiol. 2006, 127, 133-144.

(23) Søgaard, R.; Alsterfjord, M.; MacAulay, N.; Zeuthen, T. Pflugers Arch. Eur. J. Phy. 2009, 458, 733-743.

(24) Ortiz-Ramirez, C.; Mora, S. I.; Trejo, J.; Pantoja, O. J. Biol. Chem. 2011, 286, 31113-31122.

(25) Siewe, R. M.; Weil, B.; Burkovski, A.; Eikmanns, B. J.; Eikmanns, M.; Krämer, R. J. Biol. Chem. 1996, 271, 5398-5403.

(26) Meier-Wagner, J.; Nolden, L.; Jakoby, M.; Siewe, R.; Krämer, R.; Burkovski, A. Microbiol. 2001, 147, 135-143.

(27) Walter, B.; Kuspert, M.; Ansorge, D.; Krämer, R.; Burkovski, A. J. Bacteriol. 2008, 190, 2611-2614.

(28) Westhoff, C. M.; Ferreri-Jacobia, M.; Mak, D.-O. D.; Foskett, J. K. J. Biol. Chem. 2002, 277, 12499-12502.

(29) Ludewig, U. J. Physiol. 2004, 559, 751-759.

(30) Bakouh, N.; Benjelloun, F.; Hulin, P.; Brouillard, F.; Edelman, A.; Chérif-Zahar, B.; Planelles, G. J. Biol. Chem. 2004, 279, 1597515983.

(31) Javelle, A.; Lupo, D.; Li, X.-D.; Merrick, M.; Chami, M.; Ripoche, P.; Winkler, F. K. J. Struct. Biol. 2007, 158, 472-481.

(32) Bostick, D. L.; Brooks, C. L., III PLoS Comput. Biol. 2007, 3, e22.

(33) Bostick, D. L.; Brooks, C. L., III Biophys. J. 2007, 92, L103L105.

(34) Javelle, A.; Lupo, D.; Zheng, L.; Li, X.-D.; Winkler, F. K.; Merrick, M. J. Biol. Chem. 2006, 281, 39492-39498.

(35) Javelle, A.; Lupo, D.; Ripoche, P.; Fulford, T.; Merrick, M.; Winkler, F. K. Proc. Natl. Acad. Sci. U.S.A. 2008, 105, 5040-5045.

(36) Luzhkov, V. B.; Almlåf, M.; Nervall, M.; Åqvist, J. Biochemistry 2006, 45, 10807-10814.

(37) Nygaard, T. P.; Rovira, C.; Peters, H.; Jensen, M. Ø. Biophys. J. 2006, 91, 4401-4412.

(38) Liu, Y.; Hu, X. J. Phys. Chem. A 2006, 110, 1375-1381. 
(39) Lin, Y.; Cao, Z.; Mo, Y. J. Am. Chem. Soc. 2006, 128, 1087610884.

(40) Cao, Z.; Mo, Y.; Thiel, W. Angew. Chem., Int. Ed. 2007, 46, 6811-6815.

(41) Yang, H.; Xu, Y.; Zhu, W.; Chen, K.; Jiang, H. Biophys. J. 2007, 92, 877-885.

(42) Ishikita, H.; Knapp, E.-W. J. Am. Chem. Soc. 2007, 129, 12101215.

(43) Ishikita, H. FEBS Lett. 2007, 581, 4293-4297.

(44) Lin, Y.; Cao, Z.; Mo, Y. J. Phys. Chem. B 2009, 113, 4922-4929.

(45) Wang, J.; Yang, H.; Zuo, Z.; Yan, X.; Wang, Y.; Luo, X.; Jiang, H.; Chen, K.; Zhu, W. J. Phys. Chem. B 2010, 114, 15172-15179.

(46) Lamoureux, G.; Klein, M. L.; Bernèche, S. Biophys. J. 2007, 92, L82-L84.

(47) Nygaard, T. P.; Alfonso-Prieto, M.; Peters, G. H.; Jensen, M. Ø.; Rovira, C. J. Phys. Chem. B 2010, 114, 11859-11865.

(48) Akgun, U.; Khademi, S. Proc. Natl. Acad. Sci. U.S.A. 2011, 108, 3970-3975.

(49) Wang, S.; Orabi, E. A.; Baday, S.; Bernèche, S.; Lamoureux, G. J. Am. Chem. Soc. 2012, 134, 10419-10427.

(50) Hub, J. S.; Winkler, F. K.; Merrick, M.; de Groot, B. L. J. Am. Chem. Soc. 2010, 132, 13251-13263.

(51) Ullmann, R. T.; Ullmann, G. M. J. Comput. Chem. 2012, 33, $887-900$.

(52) Ullmann, G. M.; Knapp, E. W. Eur. Biophys. J. 1999, 28, 533551.

(53) Ullmann, G. M.; Kloppmann, E.; Essigke, T.; Krammer, E.-M.; Klingen, A. R.; Becker, T.; Bombarda, E. Photosynth. Res. 2008, 97, $33-53$.

(54) Klingen, A. R; Bombarda, E.; Ullmann, G. M. Photochem. Photobiol. Sci. 2006, 5, 588-596.

(55) Bashford, D. Front. Biosci. 2004, 9, 1082-1099.

(56) Gunner, M. R.; Mao, J.; Song, Y.; Kim, J. Biochim. Biophys. Acta 2006, 1757, 942-968.

(57) Antosiewicz, J. M.; Shugar, D. Mol. BioSyst. 2011, 7, 2923-2949.

(58) Becker, T.; Ullmann, R. T.; Ullmann, G. M. J. Phys. Chem. B

2007, 111, 2957-2968.

(59) Ferreira, A. M.; Bashford, D. J. Am. Chem. Soc. 2006, 128, 16778-16790.

(60) Warshel, A. Biochemistry 1981, 20, 3167-3177.

(61) Bashford, D.; Karplus, M. Biochemistry 1990, 29, 10219-10225.

(62) Calimet, N.; Ullmann, G. M. J. Mol. Biol. 2004, 339, 571-589.

(63) Bombarda, E.; Becker, T.; Ullmann, G. M. J. Am. Chem. Soc.

2006, 128, 12129-12139.

(64) Roux, B. Biophys. J. 1997, 73, 2980-2989.

(65) Azzone, G.; Benz, R.; Bertl, A.; Colombini, M.; Crofts, A.; Dilley, R.; Dimroth, P.; Dutton, P.; Felle, H.; Harold, F.; et al. BBABioenerg. 1993, 1183, 1-3.

(66) Brünger, A. T.; Karplus, M. Proteins 1988, 4, 148-156.

(67) Brooks, B. R.; Bruccoleri, R. E.; Olafson, B. D.; States, D. J.; Swaminathan, S.; Karplus, M. J. Comput. Chem. 1983, 4, 187-217.

(68) MacKerell, A. D.; Bashford, D.; Bellott, M.; Dunbrack, R. L.; Evanseck, J. D.; Field, M. J.; Fischer, S.; Gao, J.; Guo, H.; Ha, S.; et al. J. Phys. Chem. B 1998, 102, 3586-3616.

(69) Ullmann, R. T.; Ullmann, G. M. J. Phys. Chem. B 2011, 115, $10346-10359$.

(70) Bashford, D. An object-oriented programming suite for electrostatic effects in biological molecules An experience report on the MEAD project. In Scientific computing in object-oriented parallel environments; Ishikawa, Y., Oldehoeft, R., Reynders, J., Tholburn, M., Eds.; Springer: Berlin, Gemany, 1997; Vol. 1343, pp 233-240.

(71) Metropolis, N.; Rosenbluth, A. W.; Rosenbluth, M. N.; Teller,

A. H. J. Chem. Phys. 1953, 21, 1087-1092.

(72) Zwanzig, R. W. J. Chem. Phys. 1954, 22, 1420-1426.

(73) Ullmann, R. T.; Ullmann, G. M. J. Phys. Chem. B 2011, 115, 507-521.

(74) Bennett, C. H. J. Comput. Phys. 1976, 22, 245-268.

(75) Orabi, E. A.; Lamoureux, G. J. Chem. Theory Comp. 2012, 8, 182-193.
(76) Ullmann, R. T.; Ullmann, G. M. unpublished results.

(77) Bashford, D.; Case, D. A.; Dalvit, C.; Tennant, L.; Wright, P. E. Biochemistry 1993, 32, 8045-8056.

(78) te Velde, G.; Bickelhaupt, F. M.; Baerends, E. J.; Fonseca Guerra, C.; van Gisbergen, S. J. A.; Snijders, J. G.; Ziegler, T. J. Comput. Chem. 2001, 22, 931-967.

(79) Fonseca Guerra, C.; Snijders, J. G.; te Velde, G.; Baerends, E. J. Theor. Chem. Acc. 1998, 99, 391-403.

(80) Vosko, S. H.; Wilk, L.; Nusair, M. Can. J. Phys. 1980, 58, 12001211.

(81) Becke, A. D. Phys. Rev. A 1988, 38, 3098-3100.

(82) Perdew, J. P.; Yue, W. Phys. Rev. B 1986, 33, 8800-8802.

(83) Swart, M.; Th., V. D. P.; Snijders, J. G. J. Comput. Chem. 2001, $22,79-88$.

(84) Jorgensen, W. L.; Chandrasekhar, J.; Madura, J. D.; Impey, R. W.; Klein, M. L. J. Chem. Phys. 1983, 79, 926-935.

(85) Warwicker, J.; Watson, H. C. J. Mol. Biol. 1982, 186, 671-679.

(86) Gilson, M. K.; Sharp, K. A.; Honig, B. H. J. Comput. Chem. 1987, 9, 327-335.

(87) Lomize, M. A.; Lomize, A. L.; Pogozheva, I. D.; Mosberg, H. I. Bioinformatics 2006, 22, 623-625.

(88) Klapper, I.; Fine, R.; Sharp, K. A.; Honig, B. H. Proteins 1986, 1, 47-59.

(89) Beroza, P.; Fredkin, D. R.; Okamura, M. Y.; Feher, G. Proc. Natl. Acad. Sci. U.S.A. 1991, 88, 5804-5808.

(90) Rabenstein, B.; Ullmann, G. M.; Knapp, E. W. Eur. Biophys. J. 1998, 27, 626-637.

(91) Valleau, J. P.; Card, D. N. J. Chem. Phys. 1972, 57, 5457-5462.

(92) Mitchell, P. BBA-Bioenerg. 2011, 1807, 1507-1538.

(93) Rottenberg, H. BBA-Bioenerg. 1979, 549, 225-253.

(94) Skulachev, V. P. Eur. J. Biochem. 1992, 208, 203-209.

(95) Britto, D. T.; Glass, A. D.; Kronzucker, H. J.; Siddiqi, M. Y. Plant Physiol. 2001, 125, 523-526.

(96) Schmidt, I.; Look, C.; Bock, E.; Jetten, M. S. M. Microbiology 2004, 150, 1405-1412.

(97) Kashket, E. R. Annu. Rev. Microbiol. 1985, 39, 219-242.

(98) Ullmann, G. M. J. Phys. Chem. B 2000, 104, 6293-6301.

(99) Ullmann, G. M. J. Phys. Chem. B 2003, 107, 1263-1271.

(100) Bombarda, E.; Ullmann, G. M. J. Phys. Chem. B 2010, 114, 1994-2003.

(101) Huggins, D. J. J. Chem. Phys. 2012, 136, 064518.

(102) Huggins, D. J. J. Comput. Chem. 2012, 33, 1383-1392.

(103) Fadda, E.; Woods, R. J. J. Chem. Theory Comp. 2011, 7, 33913398.

(104) Kleiner, D. FEBS Lett. 1985, 187, 237-239.

(105) Gazzarrini, S.; Lejay, L.; Gojon, A.; Ninnemann, O.; Frommer, W. B.; von Wirén, N. Plant Cell 1999, 11, 937-948.

(106) Rawat, S. R.; Silim, S. N.; Kronzucker, H. J.; Siddiqi, M. Y.; Glass, A. D. M. Plant J. 1999, 19, 143-152.

(107) Pérez-Tienda, J.; Testillano, P. S.; Balestrini, R.; Fiorilli, V.; Azcón-Aguilar, C.; Ferrol, N. Fungal Genet. Biol. 2011, 48, 1044-1055.

(108) Beeder, J.; Nilsen, R. K.; Rosnes, J. T.; Torsvik, T.; Lien, T. Appl. Environ. Microbiol. 1994, 60, 1227-1231.

(109) Klenk, H.-P.; Clayton, R. A.; Tomb, J.-F.; White, O.; Nelson, K. E.; Ketchum, K. A.; Dodson, R. J.; Gwinn, M.; Hickey, E. K.; Peterson, J. D.; et al. Nature 1997, 390, 364-370.

(110) Li, X.; Jayachandran, S.; Nguyen, H.-H. T.; Chan, M. K. Proc. Natl. Acad. Sci. U.S.A. 2007, 104, 19279-19284.

(111) Lupo, D.; Li, X.-D.; Durand, A.; Tomizaki, T.; Cherif-Zahar, B.; Matassi, G.; Merrick, M.; Winkler, F. K. Proc. Natl. Acad. Sci. U.S.A. 2007, 104, 19303-19308.

(112) Ripoche, P.; Bertrand, O.; Gane, P.; Birkenmeier, C.; Colin, Y.; Cartron, J.-P. Proc. Natl. Acad. Sci. U.S.A. 2004, 101, 17222-17227.

(113) Mouro-Chanteloup, I.; Cochet, S.; Chami, M.; Genetet, S.; Zidi-Yahiaoui, N.; Engel, A.; Colin, Y.; Bertrand, O.; Ripoche, P. PLoS ONE 2010, 5, e8921.

(114) Musa-Aziz, R.; Jiang, L.; Chen, L.-M.; Behar, K.; Boron, W. J. Membr. Biol. 2009, 228, 15-31.

(115) Hall, J. A.; Kustu, S. Proc. Natl. Acad. Sci. U.S.A. 2011. 
(116) Tremblay, P.-L.; Hallenbeck, P. C. Mol. Microbiol. 2009, 71, $12-22$.

(117) McDonald, T.; Dietrich, F.; Lutzoni, F. Mol. Biol. Evol. 2011, $29,51-60$.

(118) Till, M. S.; Essigke, T.; Becker, T.; Ullmann, G. M. J. Phys. Chem. B 2008, 112, 13401-13410.

(119) Pflock, T. J.; Oellerich, S.; Southall, J.; Cogdell, R. J.; Ullmann, G. M.; Köhler, J. J. Phys. Chem. B 2011, 115, 8813-8820.

(120) Pflock, T. J.; Oellerich, S.; Krapf, L.; Southall, J.; Cogdell, R. J.; Ullmann, G. M.; Köhler, J. J. Phys. Chem. B 2011, 115, 8821-8831.

(121) Bombarda, E.; Ullmann, G. M. Faraday Discuss. 2011, 148, 173-193.

(122) Clark, T. J. Comput. Aided Mol. Des. 2010, 24, 605-611.

(123) Malde, A.; Mark, A. J. Comput. Aid. Mol. Des. 2011, 25, 1-12.

(124) Donnini, S.; Villa, A.; Groenhof, G.; Mark, A. E.; Wierenga, R. K.; Juffer, A. H. Proteins 2009, 76, 138-150.

(125) Aguilar, B.; Anandakrishnan, R.; Ruscio, J. Z.; Onufriev, A. V. Biophys. J. 2010, 98, 872-880.

(126) Wright, E.; Serpersu, E. H. J. Thermodyn. Catal. 2011, 2, 105.

(127) Aleksandrov, A.; Simonson, T. J. Comput. Chem. 2010, 31, $1550-1560$.

(128) Aleksandrov, A.; Proft, J.; Hinrichs, W.; Simonson, T. ChemBioChem 2007, 8, 675-685.

(129) Park, M.-S.; Gao, C.; Stern, H. A. Proteins 2011, 79, 304-314.

(130) Søndergaard, C. R.; Olsson, M. H. M.; Rostkowski, M.; Jensen, J. H. J. Chem. Theory Comp. 2011, 7, 2284-2295.

(131) Muddana, H.; Daniel Varnado, C.; Bielawski, C.; Urbach, A.; Isaacs, L.; Geballe, M.; Gilson, M. J. Comput. Aid. Mol. Des. 2012, 26, $475-487$.

(132) Natesan, S.; Subramaniam, R.; Bergeron, C.; Balaz, S. J. Med. Chem. 2012, 55, 2035-2047.

(133) ten Brink, T.; Exner, T. J. Comput. Aided Mol. Des. 2010, 24, 935-942.

(134) Czodrowski, P. Bioorgan. Med. Chem. 2012, doi: 10.1016/ j.bmc.2012.03.009.

(135) Donnini, S.; Tegeler, F.; Groenhof, G.; Grubmüller, H. J. Chem. Theory Comp. 2011, 7, 1962-1978.

(136) Wallace, J. A.; Shen, J. K. J. Chem. Theory Comp. 2011, 7, $2617-2629$.

(137) Goh, G. B.; Knight, J. L.; Brooks, C. L. J. Chem. Theory Comp. 2012, 8, 36-46. 